\title{
Measurement and Characterization Of the Effects of Blue Fluorescence ON Diamond APPEARANCE
}

Yun Luo, David Nelson, Troy Ardon, and Christopher M. Breeding

In this study, the effects of blue fluorescence on the appearance of diamonds-including table-down color, faceup color, brightness, and transparency - are quantitatively characterized using customized measurement systems. The results show that UV intensity in the light source affects table-down color, face-up color, and brightness of diamonds. A standardized lighting environment containing a fixed UV component is essential for accurate and consistent color evaluation that fully incorporates the effects of blue fluorescence. The "hazy" appearance that often impacts apparent diamond transparency is mainly attributed to light scattering from structural defects, but our results also indicate that strong fluorescence causes minor contrast loss in the face-up patterns of some polished diamonds. The presence of both strong fluorescence and light-scattering structural defects increases the apparent haziness. The transparency changes induced by structural defects, fluorescence, or both can be characterized by a bulk contrast evaluation method using the diamond's face-up pattern. Fluorescence is an intrinsic property of diamond that can improve the color of some stones when they are exposed to a lighting environment with significant UV content, such as daylight, but fluorescence alone does not noticeably reduce transparency.

F luorescence in diamond is a subject that has generated lively discussion in the trade for decades. It is believed that diamonds with $\mathrm{D}$ to F color grades (i.e., colorless), which do not possess enough color to offset the presence of fluorescence, are prone to appear hazy when combined with strong or very strong blue fluorescence. Lower-color diamonds with medium to very strong blue fluorescence may appear up to one color grade better due to the blue fluorescence "neutralizing" the yellow color. The trade has expressed concerns that the true color of diamonds is not represented under light containing $\mathrm{UV}(<400 \mathrm{~nm})$ and considers diamonds color graded under such light as over-graded (Cowing, 2010). Conversely, some global markets believe that fluorescence may have a beneficial impact on color and thus lower-color diamonds with fluorescence will sell at a slight premium (table 1). These mixed perceptions on the effect of fluorescence on overall diamond appearance have impacted the value of dia-

See end of article for About the Authors and Acknowledgments.

Gems \& Gemology, Vol. 57, No. 2, pp. 102-123,

http://dx.doi.org/10.5741/GEMS.57.2.102

(C) 2021 Gemological Institute of America

monds with fluorescence since the late 1970s but have been amplified in recent years (table 1).

Based on visual observations under different lighting conditions, several studies have investigated the effect of blue fluorescence on the color and overall appearance of diamonds. Moses et al. (1997) concluded that strongly blue fluorescent diamonds were per-

\section{In Brief}

- Fluorescence is an important property of gem diamonds that impacts their value and pricing in the marketplace.

- Analysis indicates that a gem diamond's visible color is only occasionally impacted in those with strong to very strong fluorescence and depends on the amount of UV in the light source.

- Modulation transfer function (MTF) is a very useful tool for examining contrast loss as a measurement of haziness in gem diamonds.

- The hazy appearance sometimes observed in gem diamonds is primarily caused by atomic-scale light scattering defects or nano-inclusions. Strong fluorescence by itself does not cause haziness, but it may produce more contrast loss in some diamonds that already have a hazy appearance. 

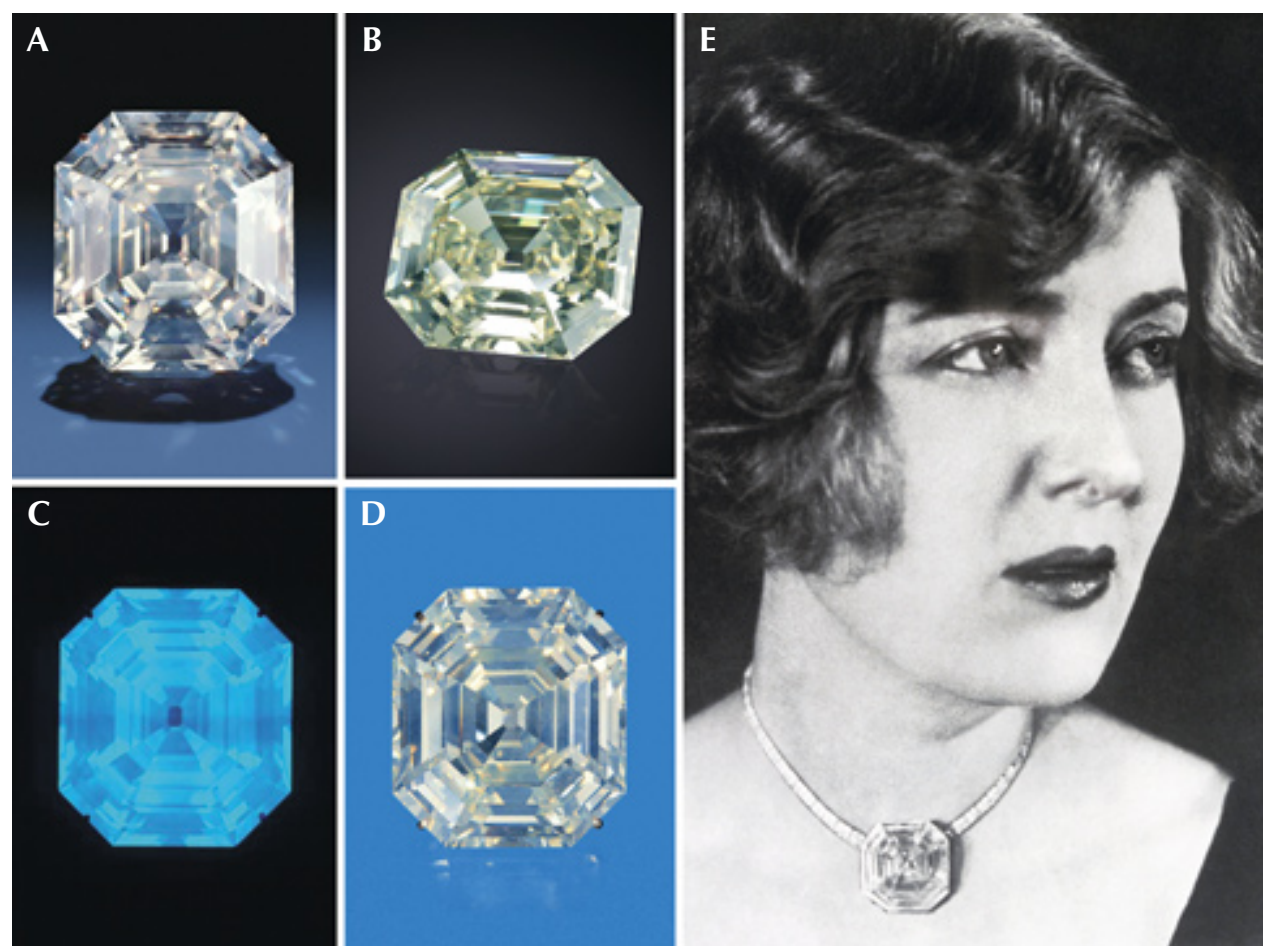

Figure 1. The $127.01 \mathrm{ct}$ Portuguese diamond was graded by GIA as $M$ color and $V S_{1}$ clarity $(A, B)$ with very strong blue fluorescence (C); photos courtesy of the Smithsonian Institution. The Portuguese diamond has been cited as a classic example of a stone being "over blue" and exhibiting a noticeably oily or hazy appearance (D). It is also shown being worn by American actress Peggy Hopkins Joyce (E). Photos by NMNH Photo Services (A), Chip Clark (B), and Harold e) Erica Van Pelt (D).

ceived to have a better color appearance when viewed face-up, with no discernible trend table-down, and that there was no observable relationship between fluorescence and transparency. Bouman et al. (2018) found that diamonds observed table-down in outdoor conditions showed improved color grades, whereas fluorescence did not directly correlate with the color grade in the face-up position. Despite no clear evidence of fluorescence negatively affecting diamond color and appearance in these studies, pricing adjustments based on fluorescence continue in the trade (tables 1 and 2).

Historically, three major challenges needed to be overcome in order to understand better the effect of fluorescence on diamond appearance: the lack of a standard methodology to quantify color and fluorescence, the fact that color and fluorescence correspond to a range of values, and the lack of an accepted illumination standard.

The absence of a standardized characterization system, together with the fact that both color grades and fluorescence evaluations correspond to a range of measurable values, makes consistent and accurate visual comparison difficult. For example, if we choose a high $\mathrm{K}$ color (closer to J) diamond with medium-low blue fluorescence to compare with a low $\mathrm{K}$ (closer to L) stone with medium-high blue fluorescence, the effect of medium fluorescence will be different because the two samples fall at opposite ends of continuous yet independent ranges for both color and fluorescence. Even with carefully aligned visual observations and spectroscopy-based measurement techniques for color and fluorescence, we still need image-based measurements that more accurately reflect how the stones appear in "real life" and can be directly linked to human visual perception. No previous study has utilized imaging systems to demonstrate and quantify the effects of fluorescence on diamond appearance. Even if we can visually identify the effect of fluorescence on face-up color by comparing very strongly fluorescent stones against inert stones under certain lighting conditions, these differences are not readily obvious in images taken under different lighting environments.

Figure 1 shows the famous $127.01 \mathrm{ct}$ Portuguese diamond, housed at the Smithsonian Institution. It has been cited as a classic example of a very strong blue fluorescent diamond displaying a noticeably oily or hazy appearance. Photos of this legendary diamond taken under different lighting setups show various aspects of its appearance, including clarity, color, and haziness. It is not easy to find the right combination of illumination and camera resolution to accurately and precisely display these effects with sufficient reproducibility.

The last but perhaps most important challenge is the standardization of illumination for color measurement and visual observation. In King et al. (2008), the GIA team concluded that a standard light source for diamond color grading should possess key daylight elements-including a UV component to truly and accurately represent how a diamond appears to 
TABLE 1. Approximate percentage pricing differential from nonfluorescent diamonds.

\begin{tabular}{|c|c|c|c|c|c|c|c|}
\hline \multirow{3}{*}{$\begin{array}{l}\text { Color } \\
\text { Grade }\end{array}$} & \multirow{3}{*}{$\begin{array}{c}\text { FL Intensity } \\
\text { (blue) }\end{array}$} & \multicolumn{6}{|c|}{ Clarity Grade } \\
\hline & & \multicolumn{2}{|c|}{ IF to VVS } & \multicolumn{2}{|c|}{ VS } & \multicolumn{2}{|c|}{$\mathrm{SI}$} \\
\hline & & 2017 & 2020 & 2017 & 2020 & 2017 & 2020 \\
\hline \multirow{4}{*}{ D-F } & Very Strong & -10 to $-15 \%$ & $\mathrm{n} / \mathrm{a}$ & -6 to $-10 \%$ & $\mathrm{n} / \mathrm{a}$ & 0 to $-3 \%$ & n/a \\
\hline & Strong & -7 to $-10 \%$ & -18 to $-25 \%$ & -3 to $-5 \%$ & -12 to $-22 \%$ & 0 to $-1 \%$ & -8 to $-16 \%$ \\
\hline & Medium & -3 to $-7 \%$ & -14 to $-21 \%$ & -1 to $-2 \%$ & -9 to $-17 \%$ & 0 & -6 to $-13 \%$ \\
\hline & Faint & $-1 \%$ & -9 to $-15 \%$ & $0 \%$ & -6 to $-12 \%$ & 0 & -4 to $-8 \%$ \\
\hline \multirow{4}{*}{$\mathrm{G}-\mathrm{H}$} & Very Strong & -7 to $-10 \%$ & $n / a$ & -3 to $-5 \%$ & $n / a$ & 0 & $n / a$ \\
\hline & Strong & -5 to $-7 \%$ & -13 to $-17 \%$ & -2 to $-3 \%$ & -10 to $-15 \%$ & 0 & -7 to $-11 \%$ \\
\hline & Medium & -1 to $-3 \%$ & -10 to $-14 \%$ & 0 to $-2 \%$ & -8 to $-12 \%$ & 0 & -5 to $-8 \%$ \\
\hline & Faint & $-1 \%$ & -7 to $-11 \%$ & 0 & -5 to $-9 \%$ & 0 & -3 to $-5 \%$ \\
\hline \multirow{4}{*}{$\mathrm{I}-\mathrm{K}$} & Very Strong & 0 to $+2 \%$ & $n / a$ & 0 to $+2 \%$ & $\mathrm{n} / \mathrm{a}$ & 0 to $+2 \%$ & $\mathrm{n} / \mathrm{a}$ \\
\hline & Strong & 0 to $+2 \%$ & -6 to $-12 \%$ & 0 to $+2 \%$ & -5 to $-11 \%$ & 0 to $+2 \%$ & -4 to $-10 \%$ \\
\hline & Medium & 0 to $+2 \%$ & -5 to $-9 \%$ & 0 to $+2 \%$ & -4 to $-8 \%$ & 0 to $+2 \%$ & -3 to $-7 \%$ \\
\hline & Faint & 0 & -4 to $-6 \%$ & 0 & -3 to $-5 \%$ & 0 & -2 to $-4 \%$ \\
\hline \multirow{4}{*}{ L-M } & Very Strong & 0 to $+2 \%$ & $\mathrm{n} / \mathrm{a}$ & 0 to $+2 \%$ & $\mathrm{n} / \mathrm{a}$ & 0 to $+2 \%$ & $\mathrm{n} / \mathrm{a}$ \\
\hline & Strong & 0 to $+2 \%$ & $\mathrm{n} / \mathrm{a}$ & 0 to $+2 \%$ & $n / a$ & 0 to $+2 \%$ & $n / a$ \\
\hline & Medium & 0 to $+2 \%$ & $\mathrm{n} / \mathrm{a}$ & 0 to $+2 \%$ & $\mathrm{n} / \mathrm{a}$ & 0 to $+2 \%$ & $\mathrm{n} / \mathrm{a}$ \\
\hline & Faint & 0 & $\mathrm{n} / \mathrm{a}$ & 0 & $\mathrm{n} / \mathrm{a}$ & 0 & $\mathrm{n} / \mathrm{a}$ \\
\hline
\end{tabular}

Data source: Rapaport price lists (https://www.diamonds.net/Prices/DetailedInfo.aspx) n/a: data not available from Rapaport 2020 update

the human eye. For the results of this study to ultimately have significance for the diamond trade and its practices and regulations, we require an illumination standard that includes UV content and thus accounts for the effects of fluorescence on diamond appearance.

Taking advantage of recent technological advances, the focus of this study was to use customized, imagebased measurement systems to quantitatively characterize the effect of fluorescence on the color, brightness (i.e., the internal and external white light return), and transparency of gem diamonds viewed in both table-down and face-up orientations under wellcharacterized lighting conditions. Our goal is to pro- vide a better understanding of the effect of blue fluorescence on diamond appearance and quantify it instrumentally. We believe this will help to reduce the confusion and biases in the industry and serve as a solid scientific foundation to ensure public trust with respect to diamond fluorescence.

\section{MATERIALS AND METHODS}

Polished Diamond Samples. Four sets of round brilliant diamonds, color graded by GIA as D, F, G, and H, were used to measure table-down and face-up color. Each set was assembled with the same color grade and

TABLE 2. Average price for one-carat, VS,-clarity, triple EX (a trade term for Excellent cut, polish, and symmetry), round brilliant cut diamonds with different fluorescence intensities.

\begin{tabular}{cc|cccc|cccc}
\hline $\begin{array}{c}\text { Color } \\
\text { Grade }\end{array}$ & NON & FNT & MED & STR & VST & FNT & MED & STR & VST \\
\hline D & $\$ 8613$ & $\$ 7860$ & $\$ 7425$ & $\$ 7171$ & $\$ 6748$ & $-9 \%$ & $-14 \%$ & $-17 \%$ & $-22 \%$ \\
E & $\$ 8082$ & $\$ 7463$ & $\$ 6816$ & $\$ 6389$ & $\$ 6282$ & $-8 \%$ & $-16 \%$ & $-21 \%$ & $-22 \%$ \\
F & $\$ 7689$ & $\$ 7007$ & $\$ 6527$ & $\$ 6432$ & & $-9 \%$ & $-15 \%$ & $-16 \%$ & \\
G & $\$ 6877$ & $\$ 6536$ & $\$ 6047$ & $\$ 5941$ & & $-5 \%$ & $-12 \%$ & $-14 \%$ & \\
H & $\$ 6675$ & $\$ 6220$ & $\$ 5917$ & $\$ 5544$ & $\$ 5346$ & $-7 \%$ & $-11 \%$ & $-17 \%$ & $-20 \%$ \\
I & $\$ 5705$ & $\$ 5395$ & $\$ 5226$ & $\$ 4942$ & $\$ 4628$ & $-5 \%$ & $-8 \%$ & $-13 \%$ & $-19 \%$ \\
J & $\$ 4933$ & $\$ 4314$ & $\$ 4411$ & $\$ 4209$ & & $-13 \%$ & $-11 \%$ & $-15 \%$ & \\
\hline
\end{tabular}

Data source: Diamond prices posted on Blue Nile for October 26, 2019 (www.bluenile.com) 
TABLE 3. Four sets of round brilliant diamonds examined in this study.

\begin{tabular}{|c|c|c|c|c|c|c|c|c|c|c|c|c|}
\hline $\begin{array}{l}\text { Sample } \\
\text { ID }\end{array}$ & Shape & Color & $\begin{array}{l}\text { Fluorescence } \\
\text { Intensity }\end{array}$ & $\begin{array}{l}\text { Weight } \\
\text { (ct) }\end{array}$ & Cut & Symmetry & Polish & $\begin{array}{c}\text { Table } \\
(\%)\end{array}$ & $\begin{array}{c}\text { Depth } \\
(\%)\end{array}$ & $\begin{array}{c}\text { Crown } \\
\text { Angle } \\
\left({ }^{\circ}\right)\end{array}$ & $\begin{array}{c}\text { Pavilion } \\
\text { Angle } \\
\left({ }^{\circ}\right)\end{array}$ & $\begin{array}{c}\text { Girdle } \\
\text { Thickness } \\
(\%)\end{array}$ \\
\hline x0237 & Round & D & $\mathrm{NON}$ & 0.53 & EX & EX & EX & 58 & 61.3 & 33.6 & 41.3 & 3.4 \\
\hline$x 4632$ & Round & D & FNT & 0.61 & EX & EX & EX & 59 & 61.0 & 32.4 & 41.3 & 4.4 \\
\hline x0517 & Round & D & MED & 0.74 & EX & EX & EX & 58 & 61.3 & 34.0 & 41.2 & 3.6 \\
\hline x8912 & Round & D & STR & 0.61 & EX & EX & EX & 59 & 60.9 & 33.4 & 41.4 & 3.3 \\
\hline x8789 & Round & $\mathrm{D}$ & VST & 0.63 & EX & EX & EX & 58 & 60.9 & 33.4 & 41.2 & 3.5 \\
\hline x0059 & Round & $\mathrm{F}$ & NON & 0.56 & EX & EX & EX & 58 & 61.7 & 34.9 & 41.2 & 3.4 \\
\hline x7519 & Round & $\mathrm{F}$ & FNT & 0.50 & EX & EX & EX & 57 & 61.7 & 34.7 & 41.4 & 2.8 \\
\hline x8316 & Round & $\mathrm{F}$ & MED & 0.52 & EX & EX & EX & 58 & 61.9 & 34.5 & 41.3 & 3.5 \\
\hline x7510 & Round & $\mathrm{F}$ & STR & 0.51 & EX & EX & EX & 58 & 61.9 & 34.6 & 41.3 & 3.3 \\
\hline x8814 & Round & $\mathrm{F}$ & VST & 0.62 & EX & EX & EX & 58 & 61.6 & 34.2 & 41.3 & 3.5 \\
\hline x2150 & Round & G & $\mathrm{NON}$ & 0.51 & EX & EX & EX & 57 & 62.1 & 34.5 & 41.2 & 3.6 \\
\hline x2008 & Round & G & FNT & 0.52 & EX & EX & EX & 57 & 62.3 & 35.0 & 41.2 & 3.6 \\
\hline x1977 & Round & G & MED & 0.41 & EX & EX & EX & 57 & 62.5 & 34.9 & 41.1 & 4.0 \\
\hline x2109 & Round & G & STR & 0.51 & EX & EX & EX & 57 & 62.1 & 35.0 & 41.0 & 3.7 \\
\hline x2356 & Round & G & VST & 0.50 & EX & EX & EX & 57 & 62.3 & 35.1 & 40.8 & 4.0 \\
\hline x7596 & Round & $\mathrm{H}$ & $\mathrm{NON}$ & 0.53 & EX & EX & EX & 57 & 61.6 & 34.3 & 41.0 & 3.7 \\
\hline x0271 & Round & $\mathrm{H}$ & FNT & 0.50 & EX & EX & EX & 57 & 61.5 & 34.0 & 41.3 & 3.3 \\
\hline x7635 & Round & $\mathrm{H}$ & MED & 0.51 & EX & EX & EX & 57 & 61.3 & 33.7 & 41.0 & 3.4 \\
\hline x8812 & Round & $\mathrm{H}$ & STR & 0.61 & EX & EX & EX & 58 & 61.2 & 33.9 & 41.2 & 3.5 \\
\hline x8937 & Round & $\mathrm{H}$ & VST & 0.54 & EX & EX & EX & 57 & 61.4 & 34.4 & 41.0 & 3.4 \\
\hline
\end{tabular}

similar sizes and proportions, but with fluorescence intensities varying from None to Very Strong (table 3). All faceted diamonds used in this study carried GIA reports and represented a range of diamond types.

TABLE 4. The 13 diamond plates used in this study, sorted by fluorescence intensity according to lightness values from fluorescence measurements.

\begin{tabular}{lccll}
\hline Sample ID & Thickness $(\mathbf{m m})$ & Lightness & Strain & Diamond Type \\
\hline 5358_A & 1.709 & 3 & Medium & laB \\
CBP-0368 & 4.314 & 3 & Strong & laAB (low) \\
CBP-0069 & 2.205 & 6 & Medium & laAB \\
CBP-0632 & 3.057 & 7 & Strong & laAB \\
5359_A & 0.876 & 12 & Medium & laB \\
CBP-0413 & 2.661 & 37 & Weak & laAB \\
CBP-0067 & 2.761 & 39 & None & laAB \\
CBP-0098 & 2.225 & 43 & None & laAB \\
CBP-0929 & 3.745 & 44 & Strong & laB $>A$ \\
CBP-0151 & 2.437 & 46 & None & laAB \\
CBP-0033 & 2.768 & 47 & None & laAB \\
CBP-0361 & 2.864 & 49 & Strong & laB $>A$ \\
CBP-0926 & 2.467 & 62 & Strong & laB $>A$ \\
\hline
\end{tabular}

Higher lightness value corresponds to stronger fluorescence intensity.
Diamond Plates. Eleven rough and two faceted diamonds were cut into plates with parallel windows (table 4). All samples were analyzed by ultraviolet/ visible/near-infrared (UV-Vis-NIR), Fourier-transform infrared (FTIR), and photoluminescence (PL) spectroscopy to provide additional information about the diamond type and atomic structural defects present. In addition, the birefringence of each sample was examined under crossed polarized light in the microscope to assess internal strain. Color and fluorescence intensity were characterized for correlation with the transparency and contrast studies.

Color Measurement Systems. Two customized imaging systems were set up to measure the table-down and face-up color of diamond samples in this study. The table-down color measurement system consists of a sample chamber with a rotational stage, a light integration hemisphere, a lens-camera assembly, and a light source with tunable UV content (figure 2A). The emission spectra from the light source show four steps of UV intensity $(0,25,50$, and $100 \%$ LED power with UV emission at $368 \mathrm{~nm}$, corresponding to calculated UV content of $<1,2,3$, and $5 \%$, respectively). For the purposes of this study, UV content refers to 
2A

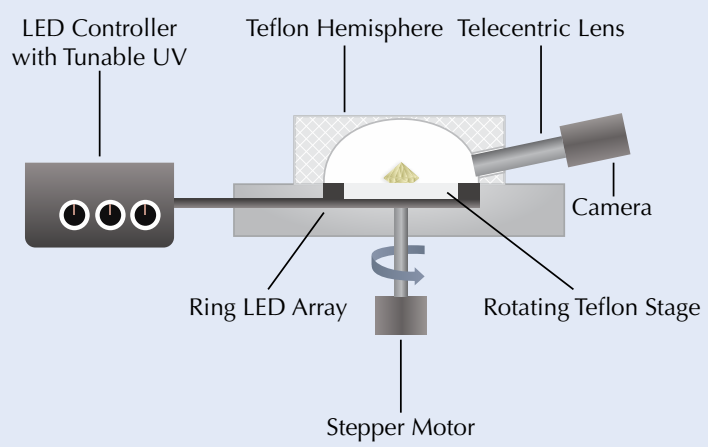

2B

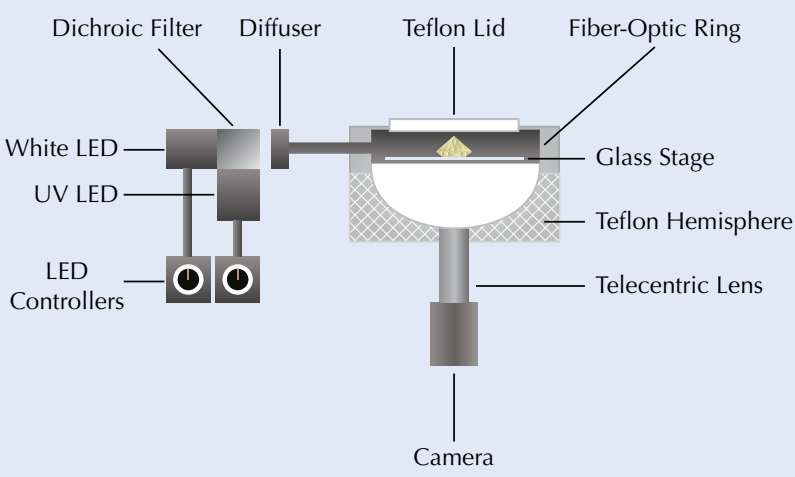

EMISSION SPECTRA
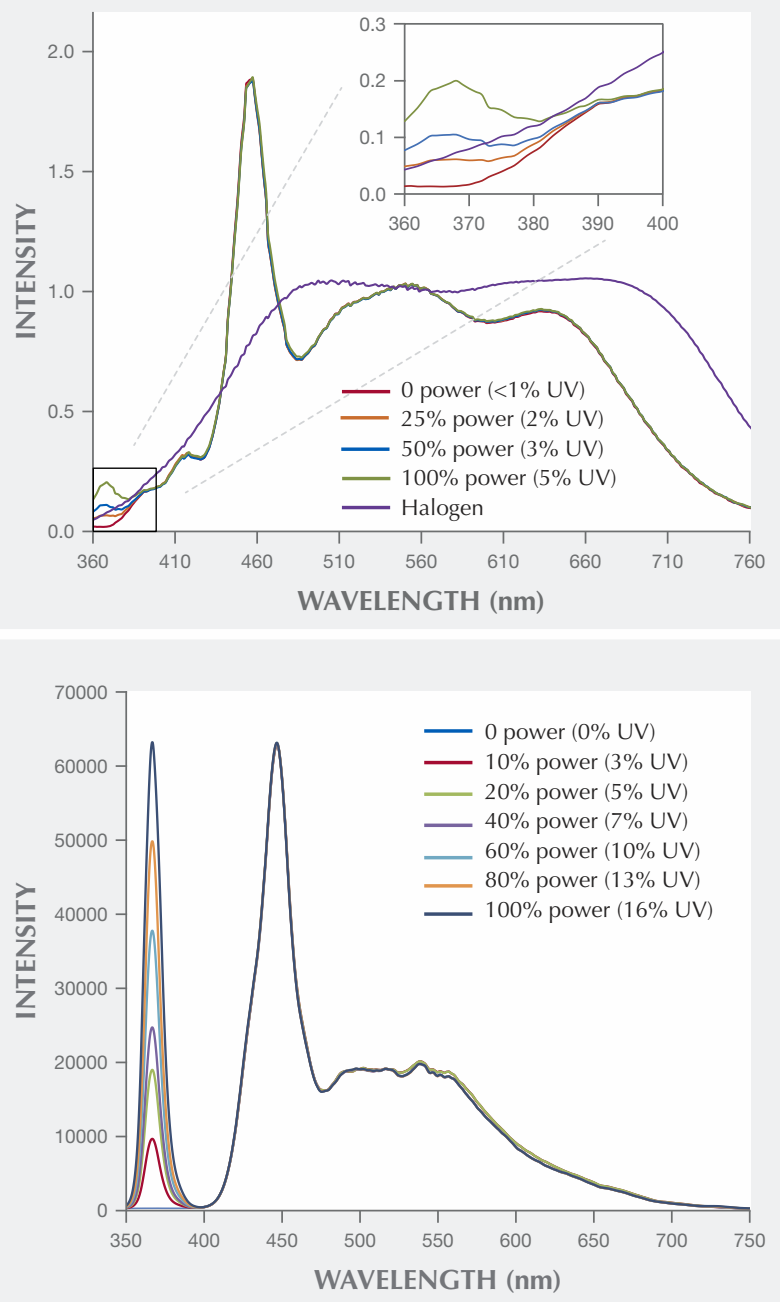

Figure 2. Color measurement experiment setups used for this study include both table-down and face-up systems. $2 A$ is the table-down color measurement system (left), together with its LED light spectra at different UV intensities compared to a halogen light source with daylight filter (right). $2 B$ shows the face-up color measurement system (left) and the spectra of the light source with different UV intensities (right).

the percentage of the total emission that occurs in the UV range (300-400 $\mathrm{nm}$ ) and is defined as follows:

$\% \mathrm{UV}=\frac{\text { area under } \mathrm{UV} \text { part of emission curve at } 300-400 \mathrm{~nm}}{\text { area under complete emission curve at } 300-850 \mathrm{~nm}} \times 100$

The face-up color measurement system was configured using a sample chamber, lens-camera assembly, and a light source with tunable UV intensity $(366 \mathrm{~nm}$ emission) at increments of $0,10,20,40,60,80$, and $100 \%$ LED power, corresponding to calculated UV content of $\sim 0,3,5,7,10,13$, and $16 \%$, respectively (figure 2B). Both of these color measurement systems were based on image processing technology. In the table-down measurement system, the numerical values of lightness, chroma, and hue $(\mathrm{LCH}$; described in box A) are used to characterize the effect of fluorescence on table-down diamond color in precise increments. Note that reported $\mathrm{LCH}$ values have been normalized to light background values. Due to camera software differences, values for hue, saturation, and lightness (see box A) were used in the face-up measurement system to correlate color grades and brightness with fluorescence.

Fluorescence Measurement System. A fluorescence measurement system consisting of a sample cham- 


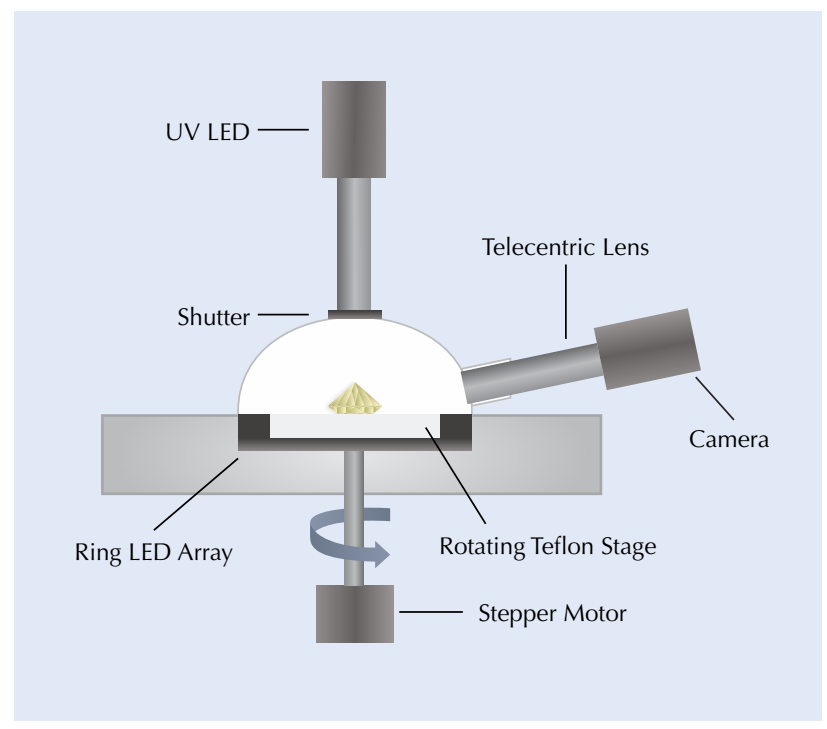

Figure 3. The fluorescence measurement experiment setup used in this study.

ber with a rotational stage, a lens-camera assembly, and a $365 \mathrm{~nm}$ UV-LED source was set up to measure the fluorescence intensity of diamond samples in this study (figure 3).
Microscope Imaging. A darkfield imaging system was designed to produce images that represent how diamonds would be viewed during grading. The system consists of a Nikon D800E, a Nikon SB-900 Speedlight, a Sigma $150 \mathrm{~mm}$ macro lens, a Stackshot macro rail package, and custom mounting fixtures. Software to control the camera and Stackshot hardware were used to automatically capture and stack a series of images for each diamond through a range of focal planes. The final processed images were used in this study to evaluate face-up appearance.

We set up two different microscopes to help understand the effect of blue fluorescence on diamond appearance under magnification. As shown in figure 4, the standard GIA microscope contains a UV component in the fluorescent overhead lamp and from the halogen bulb in the recessed light chamber used for darkfield illumination. The other testing microscope, an LED-based system, has no UV peak but does have some emissions in the wavelength range of 400 to $420 \mathrm{~nm}$ from the overhead lamp. The darkfield illumination of this testing microscope contains absolutely no UV or observable wavelengths below $420 \mathrm{~nm}$ (i.e., it is completely lacking a UV component). Photos of stones under the microscope were taken by camera through the ocular lens.

Figure 4. Light spectra collected from a standard GIA microscope overhead fluorescent lamp and darkfield illumination in the recessed light chamber (halogen) show that both contain a UV component (left). For comparison, spectra from an LED testing microscope with no UV component (either in the overhead lamp or in darkfield illumination) are shown on the right.

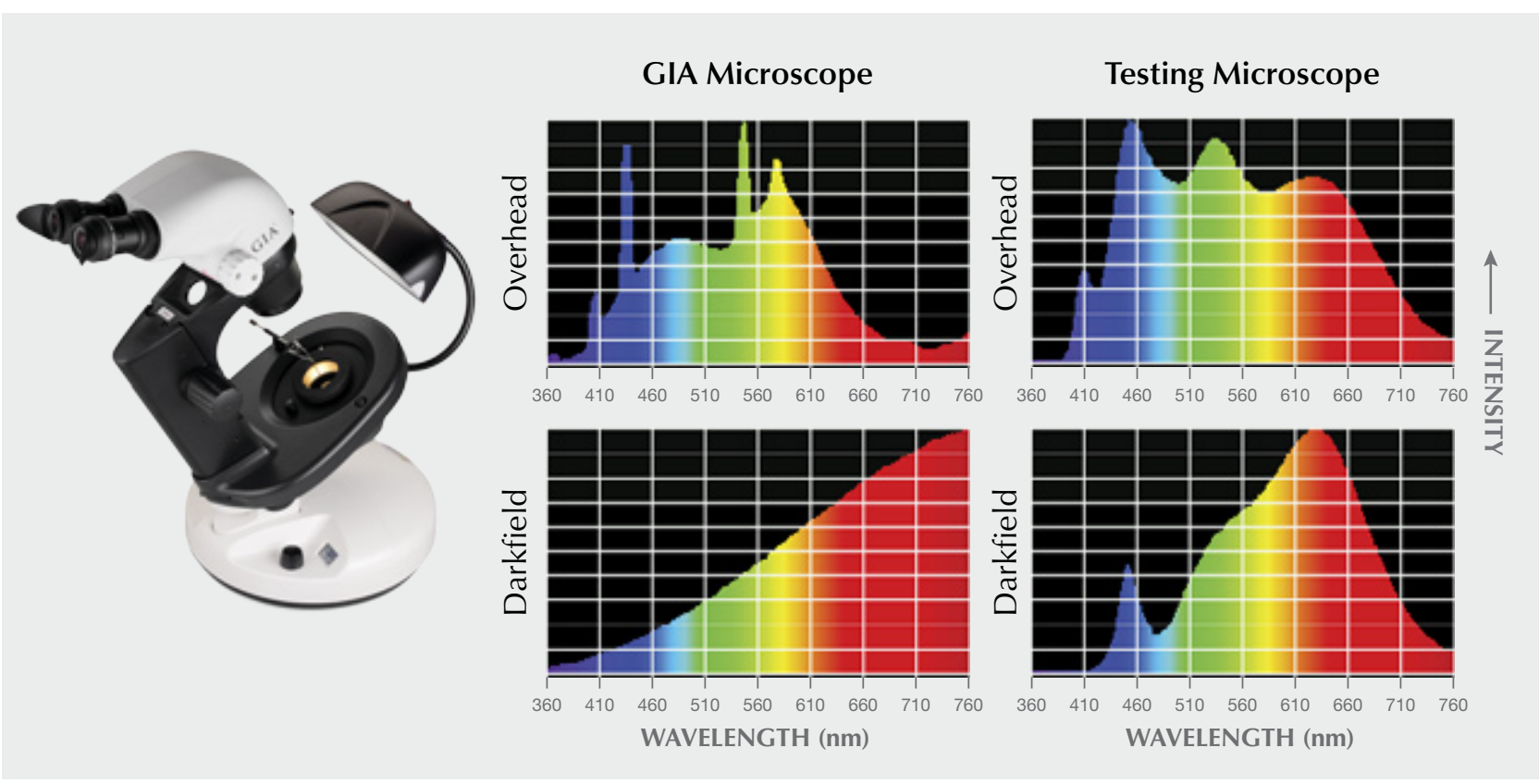




\section{Box A: Measure What You See-Color and Fluorescence}

Whenever an object is viewed, the color seen is a result of the interaction of the light source, the viewer, and the object. In attempting to achieve an accurate and consistent color and fluorescence intensity evaluation, it is important to use a standardized lighting environment that creates reproducible measurement results that characterize color and fluorescence intensity in a quantitative fashion.

Light Sources and Illuminants. A light source is a physical device that emits light with relative energy distribution in the ultraviolet to visible spectral range. It can be turned on and off and used in visual color evaluation.

The color temperature of a light source is the temperature of an ideal black-body radiator that radiates light of a color comparable to that of the light source, expressed in kelvins $(\mathrm{K})$. There are three common color temperature ranges: warm light ( $2700 \mathrm{~K}$ to $3000 \mathrm{~K}$ ), cool white $(3000 \mathrm{~K}$ to $5000 \mathrm{~K})$, and daylight (5000 K to $6500 \mathrm{~K})$.

An illuminant is an emission spectrum defined mathematically by a relative spectral power distribution that may or may not be physically achievable as a source.

By CIE definitions, the commonly used "illuminant $\mathrm{A}^{\prime \prime}$ represents incandescent light from a tungsten filament and the "illuminant $F$ " series represents a range of fluorescent lamps. Both can be readily reproduced. The D series illuminants represent natural daylight and include D65 (which refers to average zenith daylight at noon with a correlated color temperature of approximately $6500 \mathrm{~K}$ ) and D50 (which typically refers to horizon daylight in the early morning or late afternoon with a correlated color temperature of approximately $5000 \mathrm{~K}$ ). Unlike illuminant $\mathrm{A}$ and $\mathrm{F}$ series, the $\mathrm{D}$ series illuminants were defined as having specific correlated color temperature, chromaticity, and spectral power distribution, without corresponding standard light sources (visit https://cie.co.at/ for more information). Although natural daylight is commonly referred to as a standard light, the appearance and spectral characteristics of natural daylight are affected by various conditions such as the season, time of day, atmospheric conditions, and altitude. All of these factors affect how we can accurately and consistently evaluate color appearance under natural daylight. Thus, the use of simulated daylight has become the standard and accepted method for accurate color quality evaluations in many industries such as paints, plastics, textiles, graphic arts, and computer technology. It is important to note that various common sources available in the market to simulate daylight may be quite different in spectral power distribution from each other and even from any real natural daylight. Sunlight at the earth's surface typically has a UV component between 3\% and 5\% (calculated from "Reference Solar Spectral Irradiance: Air Mass 1.5," National Renewable Energy Laboratory, www.nrel.gov/grid/solarresource/spectra-am1.5.html). Therefore, a standardized daylight spectrum containing a UV component is essential to providing realistic, accurate, and consistent color and fluorescence intensity evaluations for diamond.

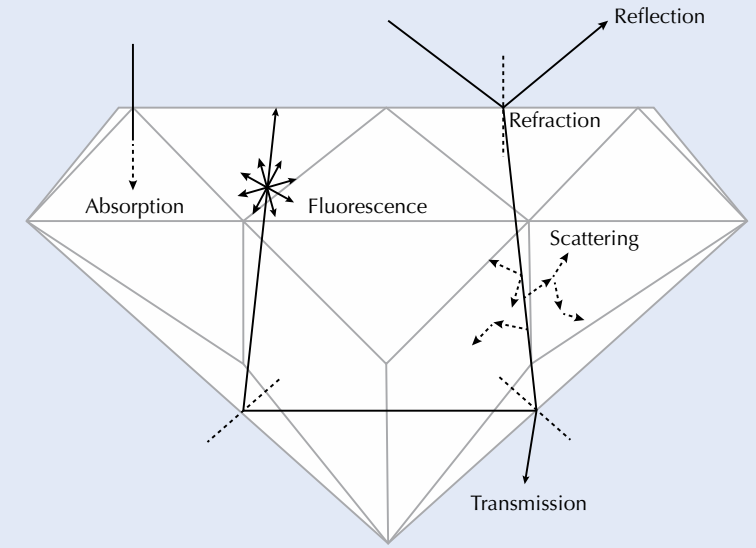

Figure A-1. Light can interact with a faceted diamond in several different ways, as shown here.

Light Interaction with Diamonds. When light strikes a diamond, a small fraction of the light is reflected while the rest is transmitted through the stone. As the light passes through, it may be refracted, absorbed, or scattered by atomic-scale defects in the diamond structure or inclusions, and it can be internally reflected by specially arranged facets in a well-cut diamond. Ultimately, the resultant wavelengths of light that exit the stone and return to the observer create the color of the diamond (figure A1). Scattering occurs when the transmitted and internally reflected light interacts with microscopic foreign particles or atomic-scale structural imperfections, causing the light to change directions. In most cases, scattering is thought to be responsible for an apparent reduction in contrast and a hazy appearance. When the particulate matter that is often responsible for light scattering is the same size or smaller than the wavelength of light in the visible range (400-700 nm), the scattering will cause a milky opalescence phenomenon known as the Tyndall effect. While scattering does affect appearance, absorption of the light by atomic-scale defects tends to have the greatest impact on diamond color by preventing particular wavelengths from being transmitted to our eyes. Additionally, when the UV component of light is absorbed by certain diamond defects, additional light of a different wavelength in the visible range is often emitted, and this is known as fluorescence. In this paper, we specifically examined the impact of blue fluorescence (by far the most common fluorescence color in natural diamond) on the appearance of faceted diamonds.

Color Space. There are different color spaces defined by the color science community to communicate and express the color of objects. GIA describes color in terms of hue, tone, and saturation (King et al., 1994). Hue refers to the diamond's characteristic color, tone to the color's relative lightness or darkness, and saturation to the color's depth or strength. In this study, we use $\mathrm{CIE} \mathrm{L}^{\star} \mathrm{C}^{\star} \mathrm{H}$ color space (figure 


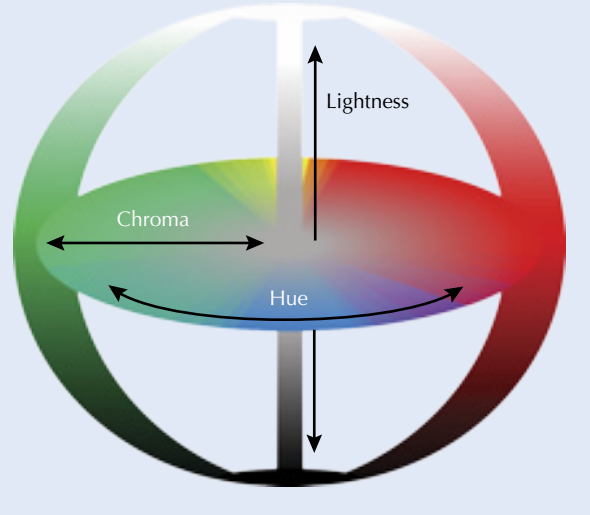

Figure A-2. Conventional color space includes three attributes: lightness, chroma, and hue.

A-2) according to GIA convention to evaluate color attributes and accurately express color and fluorescence intensity measurements in numerical terms. In this color space, L indicates lightness (also referred to as tone), C represents chroma (also known as saturation), and $\mathrm{H}$ is the hue.

Lightness (tone): The attribute by which a perceived color is judged to be equivalent to one of a series of grays ranging from black to white (ASTM, 1996).

Chroma (saturation): The attribute used to indicate the color's degree of departure from a gray of the same lightness (ASTM, 1996). It typically refers to a color's purity, intensity, or saturation.

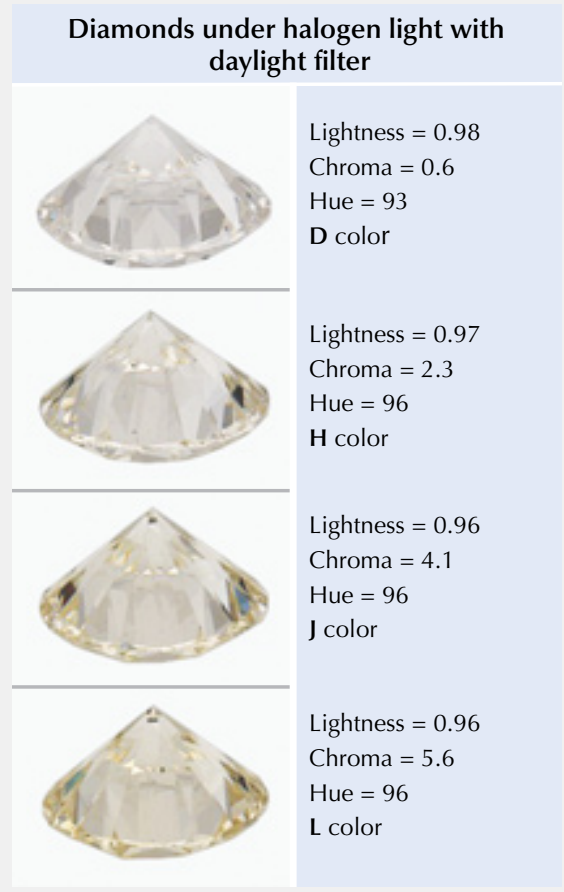

Figure A-3.

Examples of color measurements on a set of GIA research stones show the variance in color space attributes with color grades.
Hue: The attribute of color perception by means of which a color is judged to be red, orange, yellow, green, blue, purple, or intermediate between adjacent pairs of these, considered in a closed ring (ASTM, 1996).

Due to system limitations, HSL color space was used for color evaluation in the face-up measurement system. While broadly comparable to $\mathrm{LCH}$, the HSL color space consists of $\mathrm{H}$ indicating hue angle, $\mathrm{S}$ representing saturation, and $\mathrm{L}$ indicating lightness $(\mathrm{H}$ and $\mathrm{L}$ are defined above, while S correlates with chroma).

Color and Fluorescence Measurements. To help readers better understand the correlation of color terminology with the visual appearance of diamonds, figure A-3 shows images of four GIA research stones with numerical values for lightness, chroma, and hue using the color measurement experiment setup with a halogen light source with daylight filter. All of the research stones are in the D-to-Z yellow hue range, and chroma measurements are indicative of the GIA color grades $\mathrm{D}, \mathrm{H}, \mathrm{J}$, and $\mathrm{L}$.

Figure A-4 shows a different set of four research stones under UV light (365 nm). Their blue fluorescence intensities are indicated by the lightness measurement under UV, which corresponded to Faint and Strong fluorescence descriptions as reported by GIA. These are useful examples to show that each fluorescence description covers a range of actual fluorescence intensities. Samples $\mathrm{A}$ and $\mathrm{B}$ are both described as having Faint fluorescence, while samples C and D are reported as Strong, despite the obvious differences in lightness and apparent fluorescence intensities.

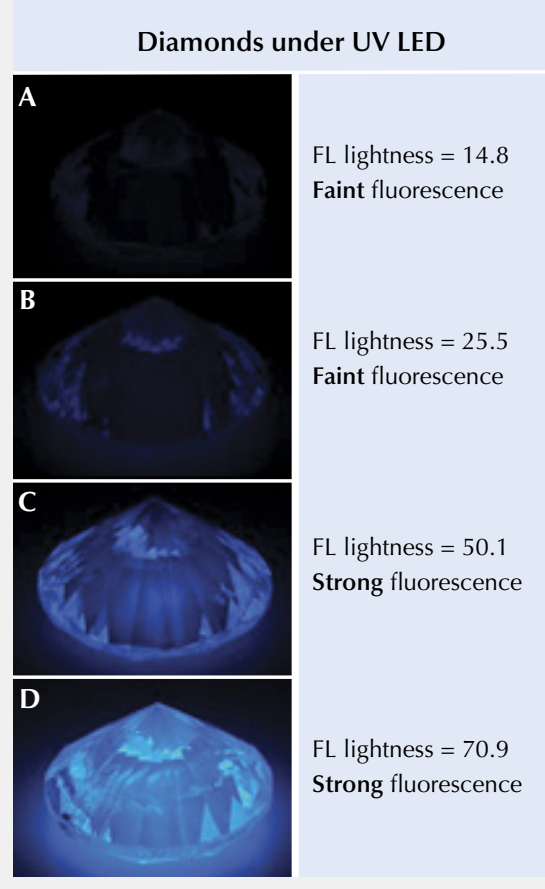

Figure A-4. Fluorescence measurements on a set of GIA research stones show the correlation between fluorescence intensity and lightness. 


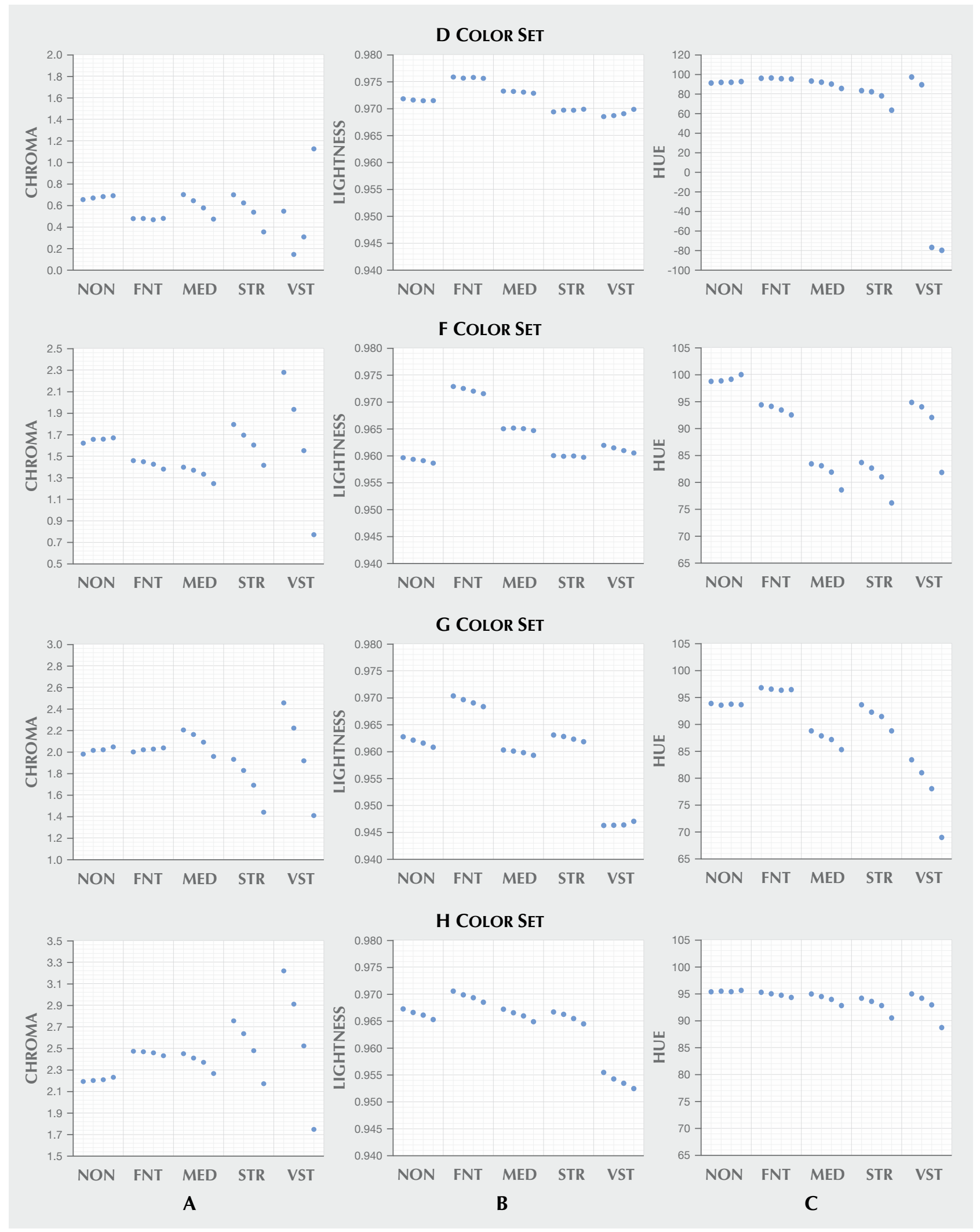

Figure 5. Trends of chroma, lightness, and hue can be seen under different UV intensities using the table-down color measurement system on $D, F, G$, and $H$ color sample sets. The four consecutive data points in each color and fluorescence value (from left to right in each grouping) represent data collected at UV increments of $<1 \%, 2 \%, 3 \%$, and $5 \%$. 


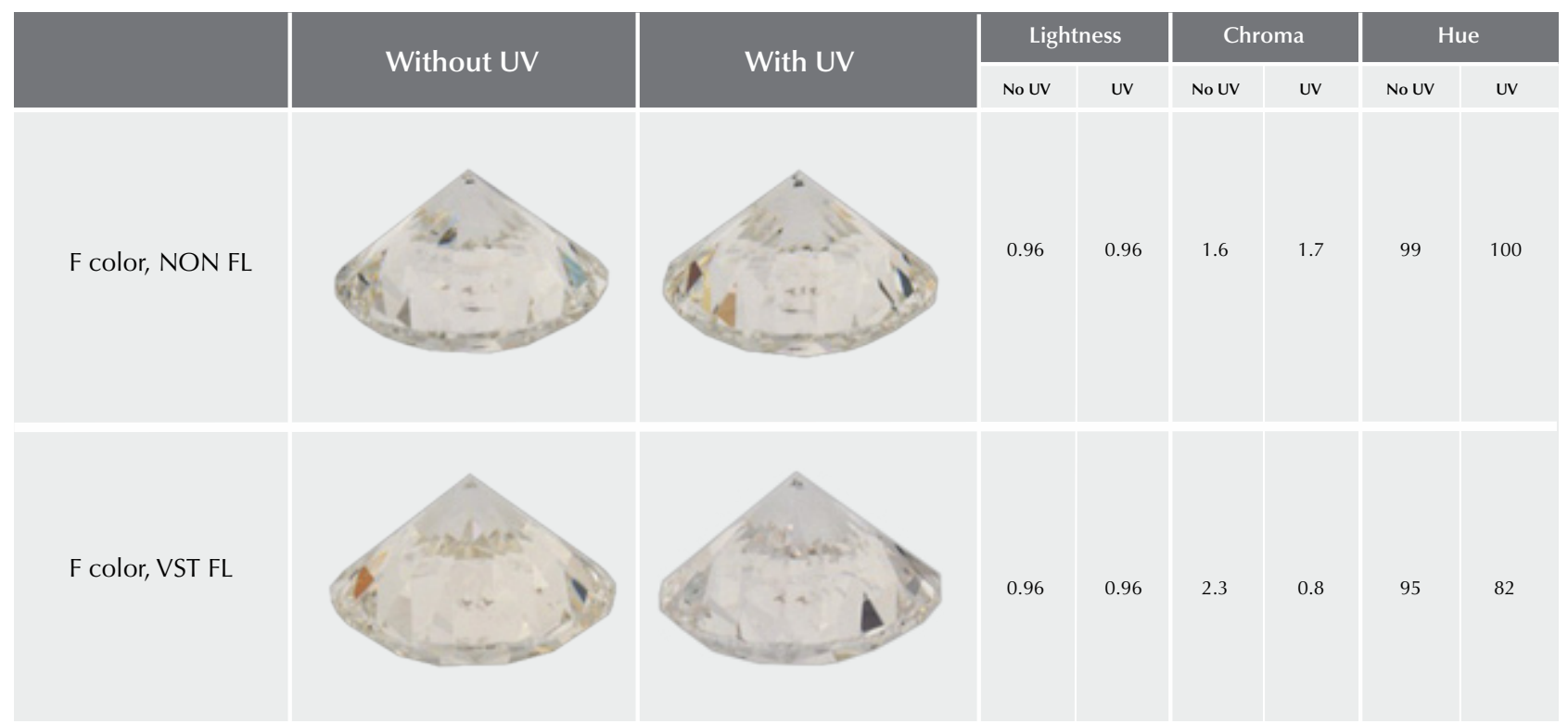

Figure 6. Stones in the F color set with fluorescence descriptions of None and Very Strong are seen without UV and with high UV intensities from the table-down color measurement system, along with corresponding attributes.

Modulation Transfer Function Measurement System. A modulation transfer function (MTF) measurement system was configured using a sample chamber, a lens-camera assembly, a light source with tunable UV content, and a micro slide with a sinusoidal array. The MTF measurement system uses image processing technology to produce percentage contrast values from the sinusoidal array target. Box B explains how MTF can be used to assess diamond transparency.

\section{RESULTS}

Table-Down Color. Similarly sized diamonds representing a cross section of color grades and fluorescence descriptions were measured on the table-down color measurement system (table 3). Each sample was measured under the same lighting environment with UV content of $<1 \%, 2 \%, 3 \%$, and $5 \%$ at 368 $\mathrm{nm}$. Figure $5 \mathrm{~A}$ shows how chroma values change with different UV intensities for D-, F-, G-, and Hcolor diamonds with fluorescence descriptions of None, Faint, Medium, Strong, and Very Strong. Diamonds with fluorescence descriptions of None or Faint show a negligible difference in chroma values as the UV content increases. Those with Medium fluorescence start to show a slight decrease in chroma values as UV content increases. Diamonds with Strong or Very Strong fluorescence descriptions show obvious reductions in chroma values with increasing UV content. The color can change as much as two color grades for diamonds with Very Strong fluorescence as UV content increases from $<1 \%$ to
$5 \%$. The D-color diamond with Very Strong fluorescence shows a sharp reduction in chroma between $<1 \%$ and $2 \%$ UV content, followed by a sharp increase in chroma as UV content increases to $3 \%$ and $5 \%$. The reduction in chroma corresponds to a desaturation of the subtle yellow color component as it becomes "neutralized" by blue fluorescence; the increase in chroma is caused by the fluorescence emission, which begins to dominate the color and shifts the hue from yellow toward blue.

Figures 5B and 5C show how lightness and hue values for these diamonds change under different $\mathrm{UV}$ intensities. For lightness, D- and F-color diamonds do not show an obvious change as UV content increases, whereas nearly all G- and H-color diamonds show a consistent decrease in lightness values across fluorescence descriptions. Diamonds with fluorescence descriptions of None or Faint show negligible changes in hue value as UV content increases. Diamonds with Medium fluorescence start to show some change in hue value with higher UV content, and those with Strong or Very Strong fluorescence show sharp changes in hue value as UV content increases. The D-color diamond with Very Strong fluorescence shows a significant change in hue value, from 100 to -80 , as UV content increases to $3 \%$. This dramatic change in hue value represents a hue change from yellow to blue.

The diamonds in the F color set with fluorescence descriptions of None and Very Strong are shown in figure 6 in lighting environments with no UV con- 


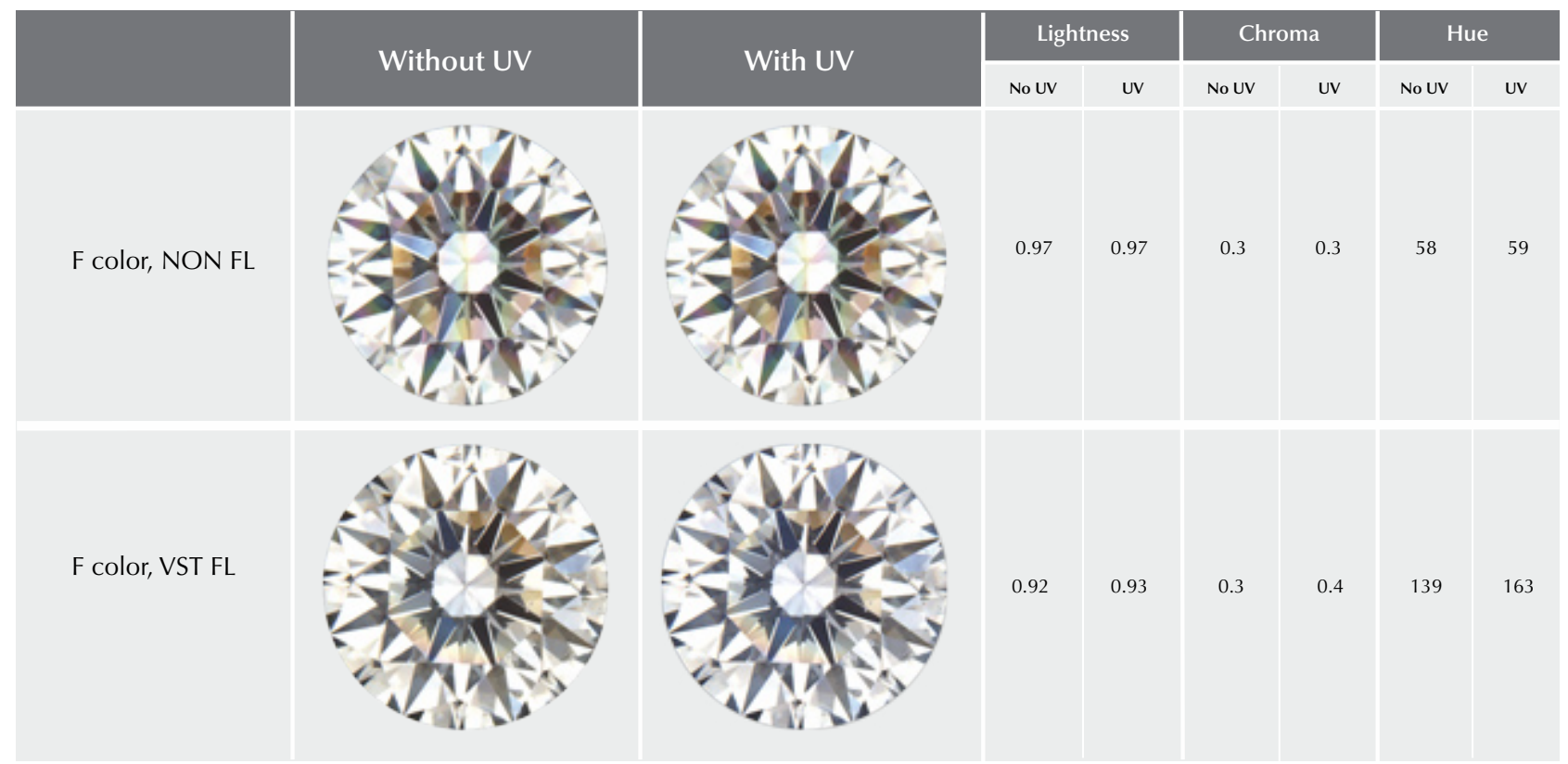

Figure 7. The same stones in the F color set from figure 6 are seen here without UV and with maximum UV intensities from the face-up imaging system, along with corresponding attributes.

tent $(<1 \%)$ and with relatively high UV content $(5 \%)$ when table-down color measurements were made. When we add UV to the lighting environment, there is no obvious color change in the diamond with a fluorescence description of None. The diamond with Very Strong fluorescence, however, shows a desaturated color as UV is added to the lighting environment. This demonstrates how blue fluorescence can neutralize a yellow color in some diamonds by the complementary color principle. The hue angle moved from normal yellow (95) to blue (82), and the chroma value decreased from 2.3 to 0.8 .

Face-Up Color. Faceted diamonds (table 3) with a range of fluorescence descriptions were imaged using light with incrementally higher UV content. For each face-up image, a square region confined to the table of the diamond was selected for measurement. The average hue, saturation, and lightness for the array of pixels in the selected area were calculated for each image. A diamond's face-up color is affected by the pattern and reflections from light return, which depend mainly on cutting parameters. Thus, the quantified hue, saturation, and lightness values in this process are not comparable to the color derived from the table-down color measurement system. Using the stone with a fluorescence description of None in each color grade as the baseline, the resulting values are compared to the level of UV content for the related images to examine the effect of UV on the face- up color and appearance of diamonds with different fluorescence descriptions.

The color of diamonds with weak or no fluorescence is shown to be unaffected by UV, while the color of diamonds with stronger fluorescence is systematically altered. The stones in the F color set with fluorescence descriptions of None and Very Strong from the face-up imaging system show that for diamonds with little to no fluorescence, the color and appearance do not change with the addition of UV, but for diamonds with fluorescence, the addition of UV causes the hue and lightness values to increase and saturation to decrease. This makes sense if we consider that the mechanism of fluorescence converts invisible UV light into visible light, thereby increasing the observed light return. Saturation values for diamonds with fluorescence are reduced with exposure to UV as the blue fluorescence neutralizes their normally yellow hue. However, if the measured hue changed from the yellow to blue range, we may see a slight increase in saturation with increasing UV (figure 7 , bottom). Our results are consistent with the faceup trends visually observed by Moses et al. (1997), which suggested that the strongly fluorescent diamonds were perceived to have a better color appearance when viewed face-up.

Transparency. Microscope Imaging. Figure 8 shows a D-color diamond with Very Strong fluorescence (left) next to a nonfluorescent D-color diamond (right) 


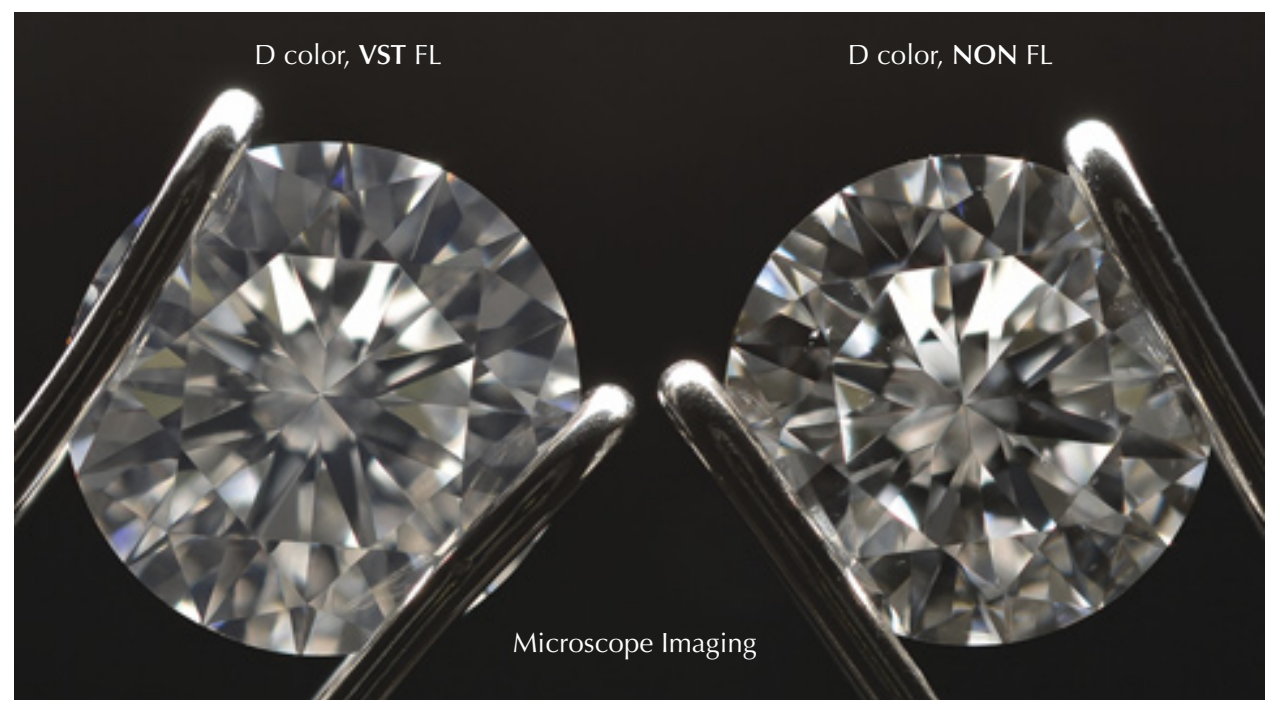

Figure 8. The stones with Very Strong fluorescence and None in the D color set are shown under the same microscope setup for transparency evaluation.

under the same lighting condition (samples from the $\mathrm{D}$ color set in table 3 ). With the same color grade and similar proportions, the diamond with Very Strong fluorescence appears slightly more "milky white" than the diamond with no fluorescence; the pattern of the diamond with Very Strong fluorescence also looks softer than the pattern in the diamond with no fluorescence, which is very crisp and sharp. We have examined many diamonds with the same color grade, similar proportions, and fluorescence descriptions ranging from None to Very Strong that do not have a hazy appearance (figure 9A). We also observed two diamonds, one with a fluorescence description of None and the other with Strong fluorescence, that showed

Figure 9. Diamond images with different degrees of haziness/transparency are plotted by fluorescence intensity.

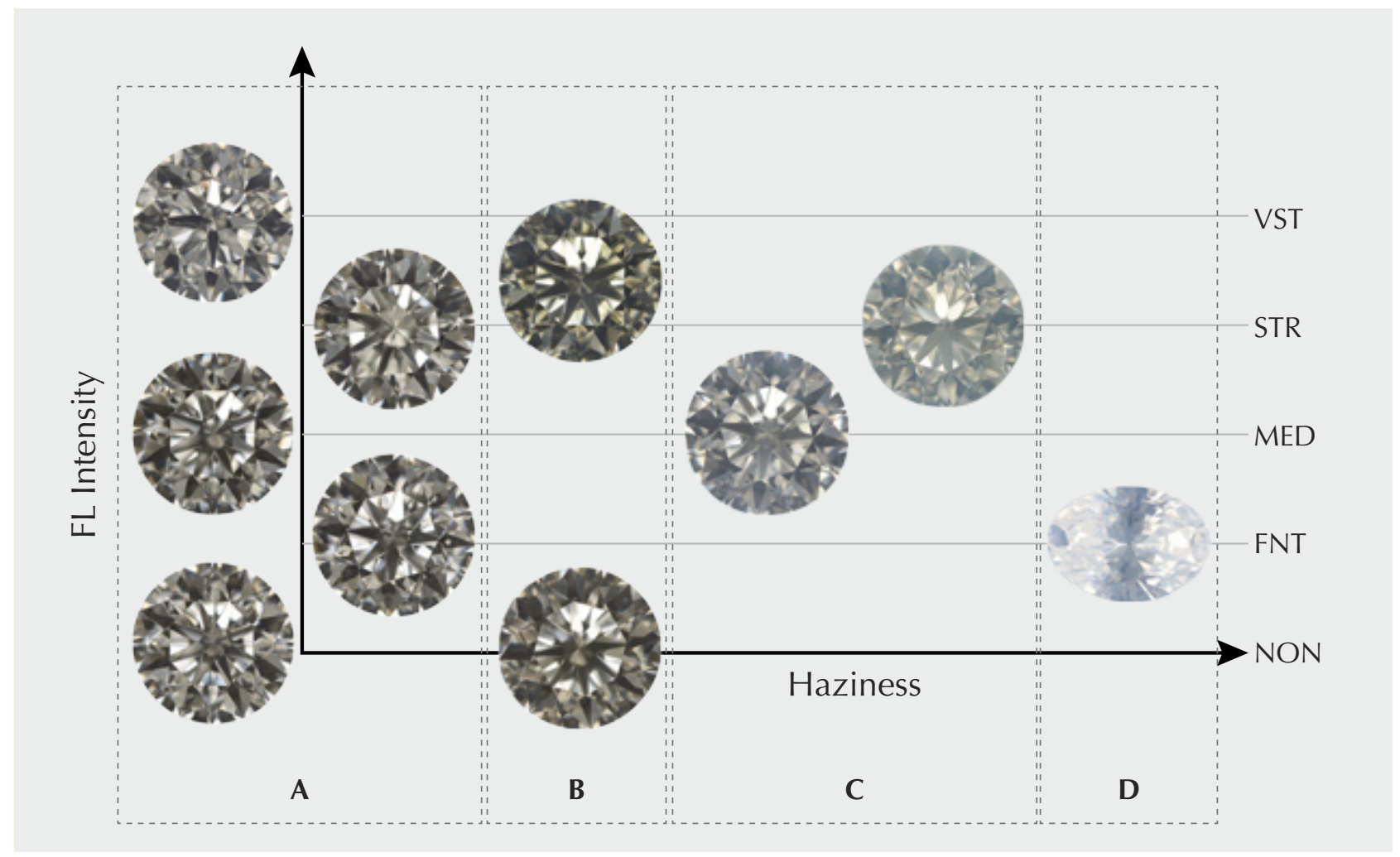



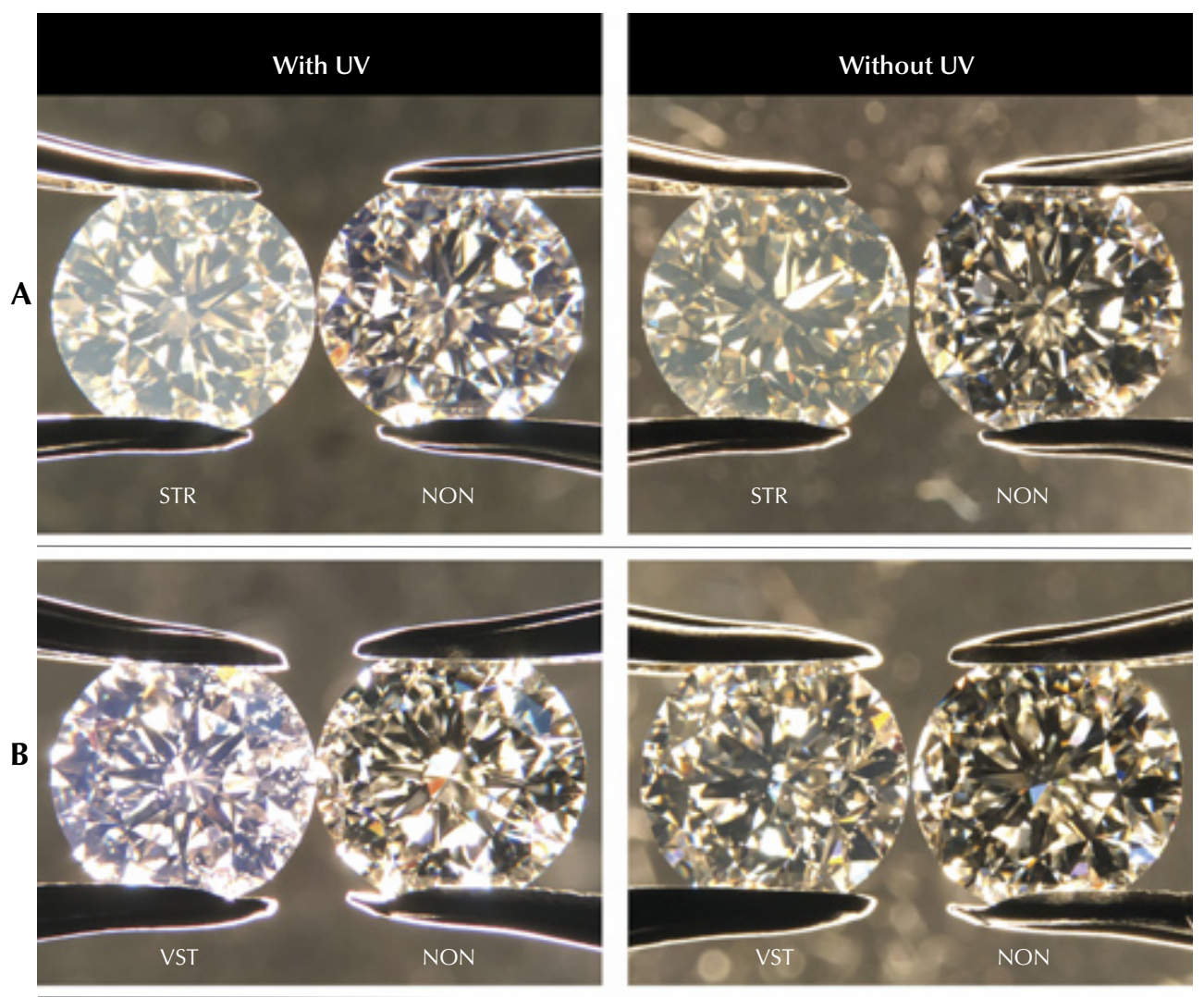

Figure 10. Sets of diamonds with different UV intensities were examined and imaged under microscope darkfield illumination, with and without UV content.
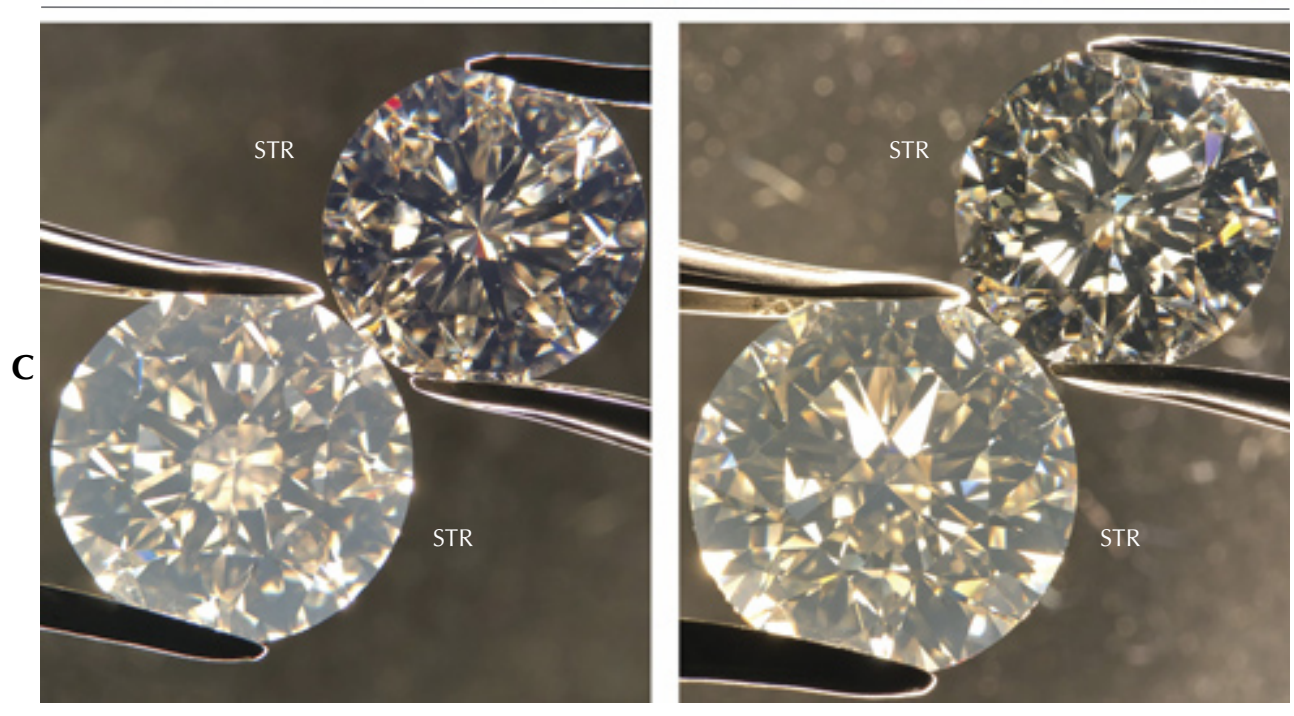

the same degree of slight haziness (figure 9B). We have seen diamonds with Medium and Strong fluorescence that have very obvious haziness (figure 9C), and a diamond with Faint fluorescence that was so milky it received a Fancy White color grade (figure 9D). Given that there is no significant correlation between the occurrence of haziness and fluorescence intensity from our observations, we believe there is no inherent link between the two.
In order to distinguish between haziness created by light scattering and contrast loss due to fluorescence, pairs of diamonds with pertinent differences were compared microscopically in two different darkfield lighting environments (figure 4): one containing UV and one without UV. With UV, the diamond with a fluorescence description of None appears quite clear, while the diamond with Strong fluorescence looks very hazy (figure 10A, left). Without UV, the diamond 


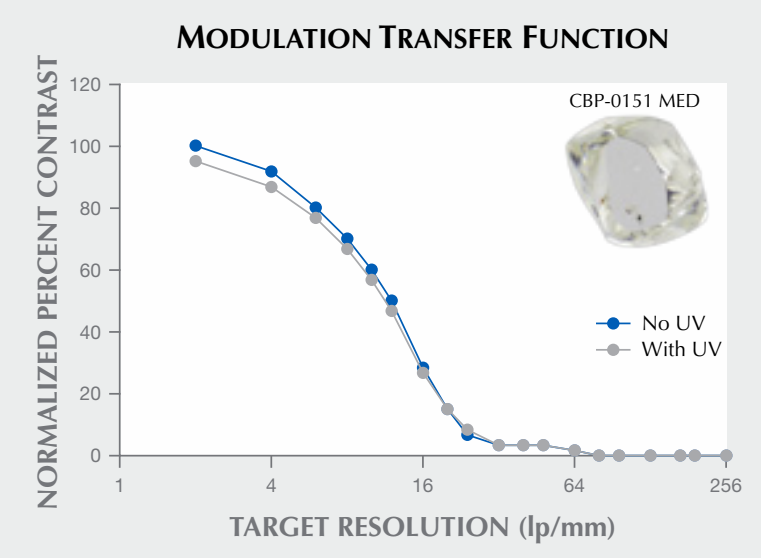

Figure 11. MTF measurement is shown for a diamond with Medium fluorescence. The reduction in percentage contrast values under UV excitation indicates contrast loss due to fluorescence.

with Strong fluorescence is still hazy, though possibly to a somewhat lesser degree (figure 10A, right). Thus, it appears that fluorescence is not the main contributor to the haziness of this diamond. Figure 10B shows two G-color diamonds with no apparent haziness. Viewed under the microscope lighting with UV content (left), it is easy to see blue luminescence from the diamond with Very Strong fluorescence. However, this diamond does not show obvious haziness under lighting environments with or without UV content. The last pair shown (figure 10C) have Strong fluorescence. Without UV, one appears clear while the other is hazy. With UV content, we observe the same thing. However, it appears that the haziness of the diamond, together with the fluorescence, makes the stone look brighter and whiter.

Modulation Transfer Function. Contrast reproduction and optical resolution of diamond samples with a range of fluorescence intensities were measured using modulation transfer function analysis as described in box B. Measurements were made while the samples were exposed to UV and while they were not exposed to UV. By comparing measurements of samples with different fluorescence intensities, the effect of fluorescence on contrast reproduction and optical resolution can be observed. For this study, contrast measurements collected with and without UV are normalized to a single maximum measured contrast.

Figure 11 shows differential percentage contrast measurements for one diamond plate with Medium fluorescence. In this figure, the $\mathrm{x}$-axis units are linepairs per millimeter and the y-axis units are normalized percentage contrast. Because the contrast values for the series collected with UV exposure are less than the contrast values for the series collected without UV exposure, a reduction in contrast due to UV exposure is measured in this fluorescent diamond plate.

The differences in contrast between measurements made with and without UV exposure for the 13 diamond plates included in this study are shown in figure 12. Fluorescent samples are shown to have reduced contrast when exposed to $U V$, while inert samples are unaffected. The summed contrast difference for each sample is plotted against fluores-

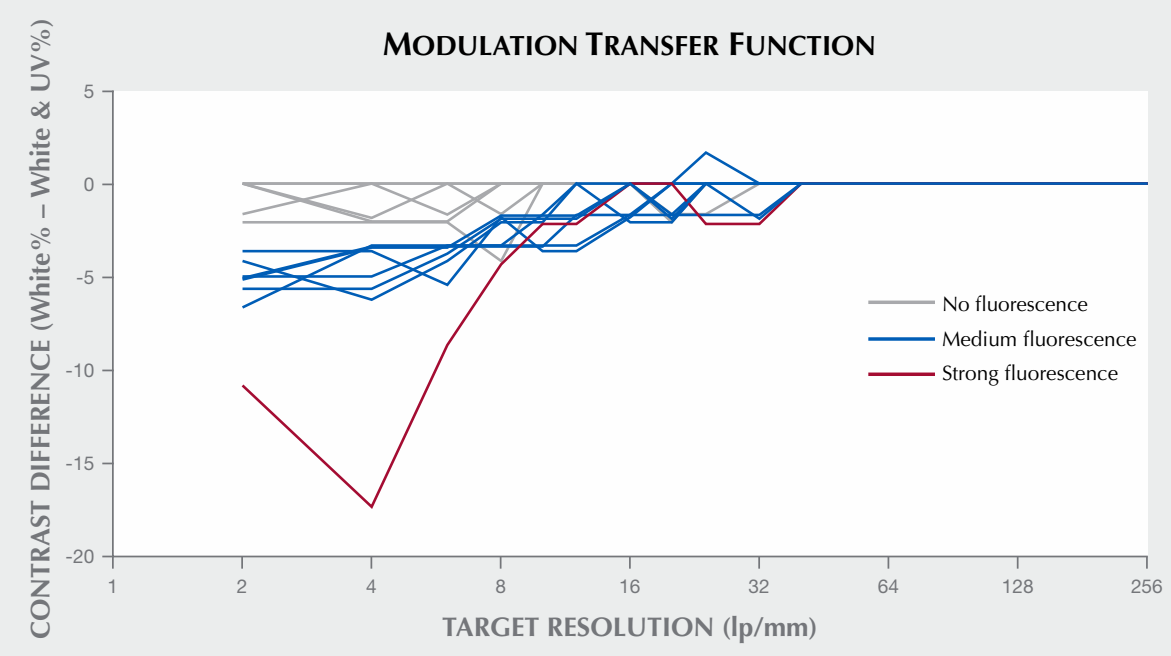

Figure 12. Contrast loss under UV excitation was determined on 13 diamond plates. 


\section{Box B: Measure What You See-Transparency}

When looking at a gem diamond, an observer usually focuses on color, clarity, and cut. Rarely does transparency come to mind, as most people assume that all gem diamonds are completely transparent. This is not always the case, however, and reduced transparency can have a significant effect on a diamond's appearance. While transparency can be assessed visually, that approach tends to be subjective and difficult to quantify. Evaluating the contrast in a stone is a more objective approach to this problem.

Transparency: The degree of regular transmission, and thus the property of a material by which objects may be seen through a sheet of that material (ASTM, 1996).

Haziness: An appearance produced by the scattering of light in a specimen, responsible for the apparent reduction in contrast of objects viewed through it (ASTM, 1996).

In optical science, a modulation transfer function (MTF) is routinely used to measure the ability of an optical component to transfer contrast at a given resolution-i.e., its optical transparency. Optical components (a lens, for example) can cause resolution and contrast to be reduced (figure B-1, top) when used in an optical system. This effect can be characterized by measuring lightness across a series of equally spaced, alternating black and white lines imaged through the lens (figure B-1, bottom) and calculating contrast as the difference in lightness between the black and white lines. Then, by comparing this contrast measurement to a maximum measurable contrast, using the formula in figure B-2, the percentage contrast can be determined. Percentage contrast measurements using a range of line-pair frequencies describe the transparency of the lens. We adapted this concept to evaluate the transparency of diamond plates with different fluorescence intensities under lighting environments with and without a UV component. An optical target from Edmund Optics with line-pair frequencies ranging from 2 to $256 \mathrm{lp} / \mathrm{mm}$ (line-pairs per millimeter) was used for this study.

Diamond Plates. MTF was used to measure the transparency of the diamond plates, which are placed between a lens-camera assembly and an optical target (figure B-3, A). An image is captured and cropped to include only the area of interest. A custom application calculates the average lightness value for each column of pixels in the image and uses those values to identify the locations of a series of local maxima and minima that correspond to the location of line-pairs in the optical target (figure B-3, B). Lightness values at these locations are paired together across each row of pixels, and their differences in value are assembled into a density plot to observe their distribution. The average value in the data set is used to calculate the normalized percentage contrast for that target (figure B-3, C). The percentage contrast values are calculated using an image of a plate taken with no UV stimulation against an image taken with UV stimulation; therefore, the plate is being measured against itself. Doing this removes concerns about path length differ-
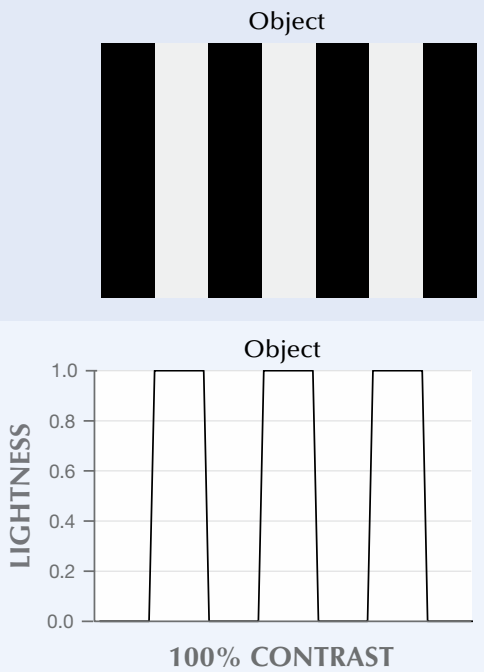
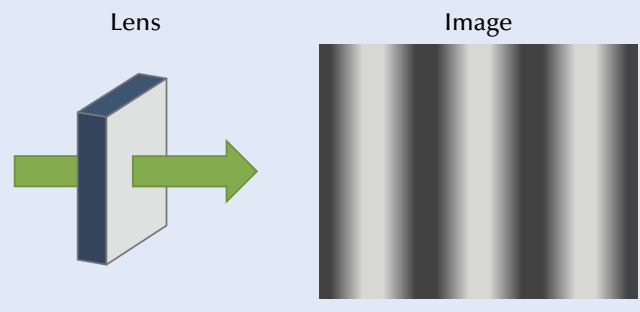

Image
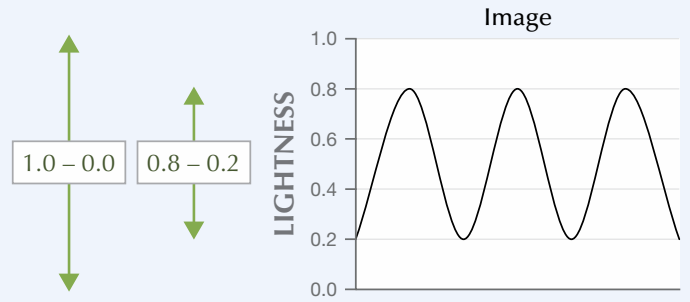

$60 \%$ CONTRAST
Figure B-1. An object imaged through a lens is shown to have reduced contrast and resolution. 
Pixels
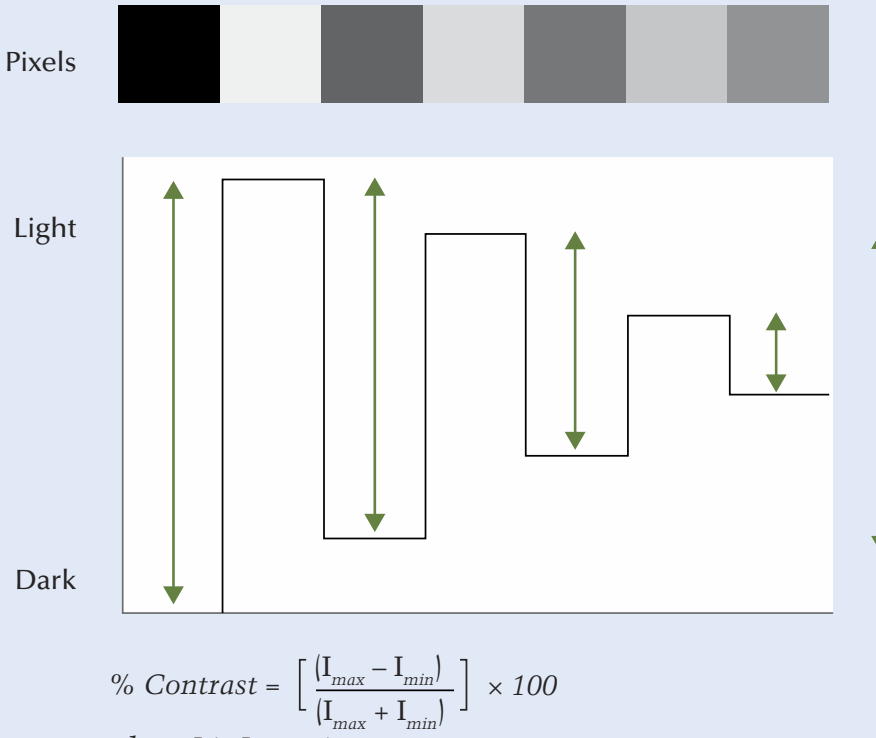

Contrast

Figure B-2. Contrast was calculated as shown here for the modulation transfer function (MTF) study.

where I is Intensity

ences, which allows us to compare bulk fluorescence values.

Polished Diamonds. Patterns of bright and dark areas produced by light interacting with the facets of a wellpolished diamond create a mechanism by which an ob- server can gauge the transparency of that diamond. When a diamond is very transparent, these patterns are perceived to be high in contrast, while for less transparent stones the perceived contrast is reduced-dark areas are less dark, bright areas less bright. The effects of poor polishing were not investigated in this study.
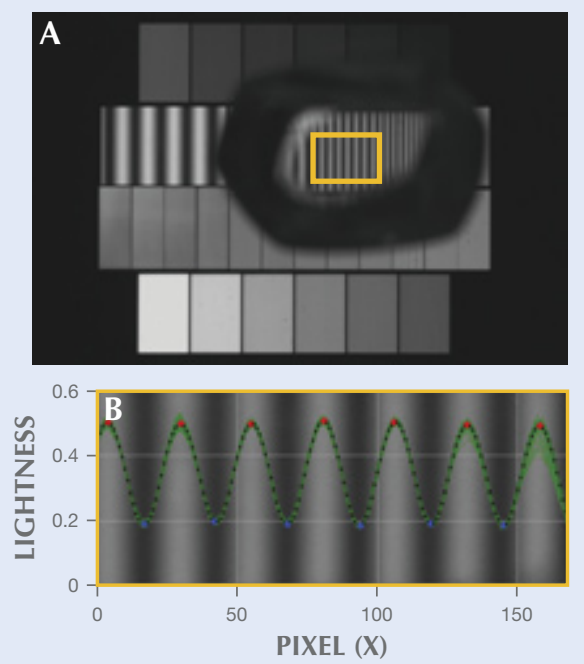

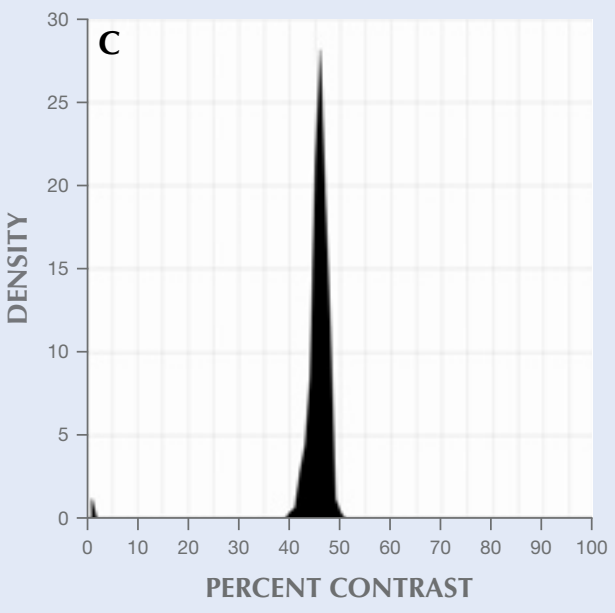

Figure B-3. An optical target is imaged through a diamond plate to conduct MTF measurements. Custom software analyzes a select area of the image and produces the percentage contrast value. 


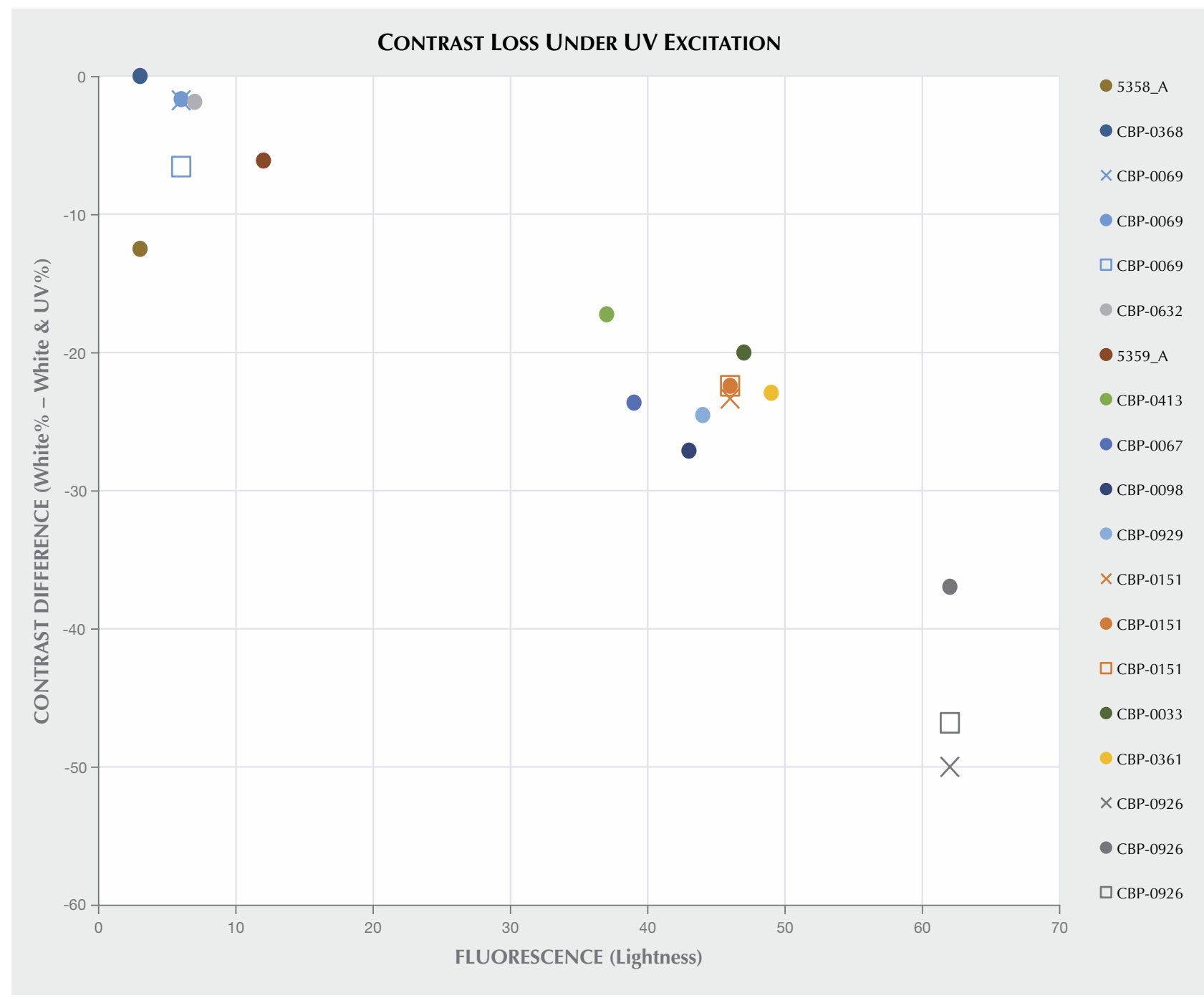

Figure 13. Correlation was observed between fluorescence intensity and contrast difference from measurements made with and without UV.

cence intensity in figure 13 and shows a correlation between these two factors. Replicated measurements for three samples are included in this figure to demonstrate measurement repeatability. The observed changes in contrast difference do not correlate with strain intensity or diamond type, strongly suggesting that the contrast loss is primarily due to fluorescence.

\section{DISCUSSION}

Illumination Standardization. Fluorescence is an intrinsic property of more than $30 \%$ of the diamonds seen by GIA's laboratory (Moses et al., 1997). Some in the diamond trade argue that because typical office lighting lacks $365 \mathrm{~nm}$ and $385 \mathrm{~nm}$ UV content, it reveals the "true color" of diamonds. Based on figure 14, we can see that office lighting does in fact contain some amount of UV light. We also need to keep in mind that blue fluorescence caused by the N3 defect can be activated by wavelengths up to $420 \mathrm{~nm}$ (Luo and Breeding, 2013). If a lighting environment includes light in the 390-420 nm range, a diamond's color will be viewed with some amount of blue fluorescence from the N3 defect. While most commercial light sources do contain light in the $400-420 \mathrm{~nm}$ range (blue light) but little in the UV range $(<400 \mathrm{~nm})$, our work investigates the effects of varying the amount of UV light (and thus the fluorescence produced) on a diamond's appearance. Consider, for example, a fluorescent lamp with no emission below $390 \mathrm{~nm}$ (figure 15). A diamond with strong fluorescence (left) glows blue 


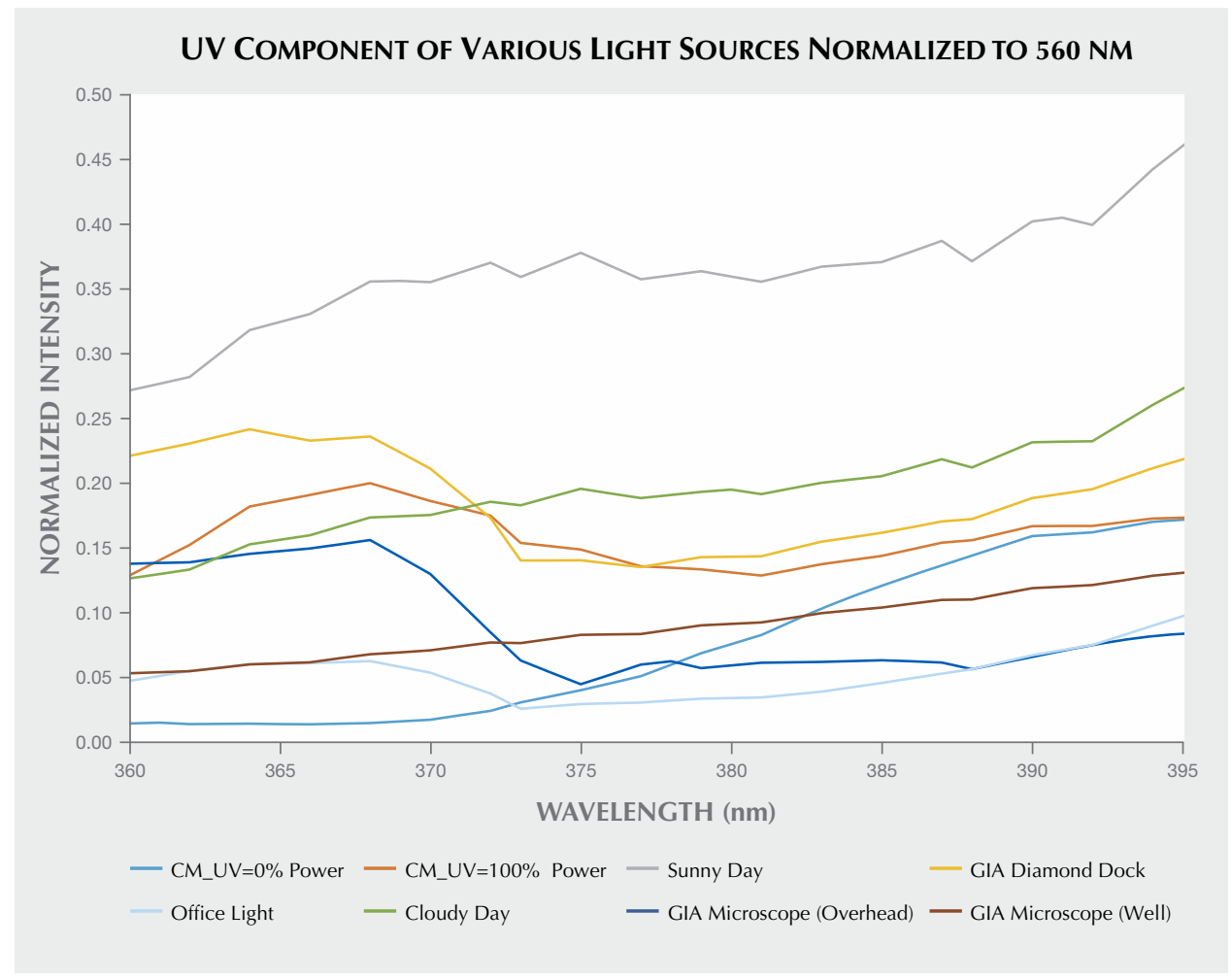

Figure 14. A comparison of the UV content of common light sources, including the color measurement system (CM) used for this study, the GIA DiamondDock, the GIA microscope overhead and well light, office light, and daylight. All spectra have been normalized at the $560 \mathrm{~nm}$ value by convention (King et al., 2008).

in this light, while a nonfluorescent diamond (right) does not. This illustrates the importance of using standardized illumination with fixed UV content to define the color grade of a diamond.

UV content is typically reported as a peak intensity output percentage normalized to a certain wavelength. However, depending on the shape and position of the UV peak, the fluorescence reaction will differ and consequently the visual appearance of the fluorescence and its effect on diamond color will also be different (Luo and Breeding, 2013). In order to consistently evaluate fluorescence and its effects on overall appearance, we again emphasize that an agreed-upon standard of illumination for color meas-

Figure 15. Two G-color stones with similar size were imaged for comparison under the overhead lamp from the testing microscope with no UV peak at $365 \mathrm{~nm}$ but with wavelength in the 390-420 nm range.

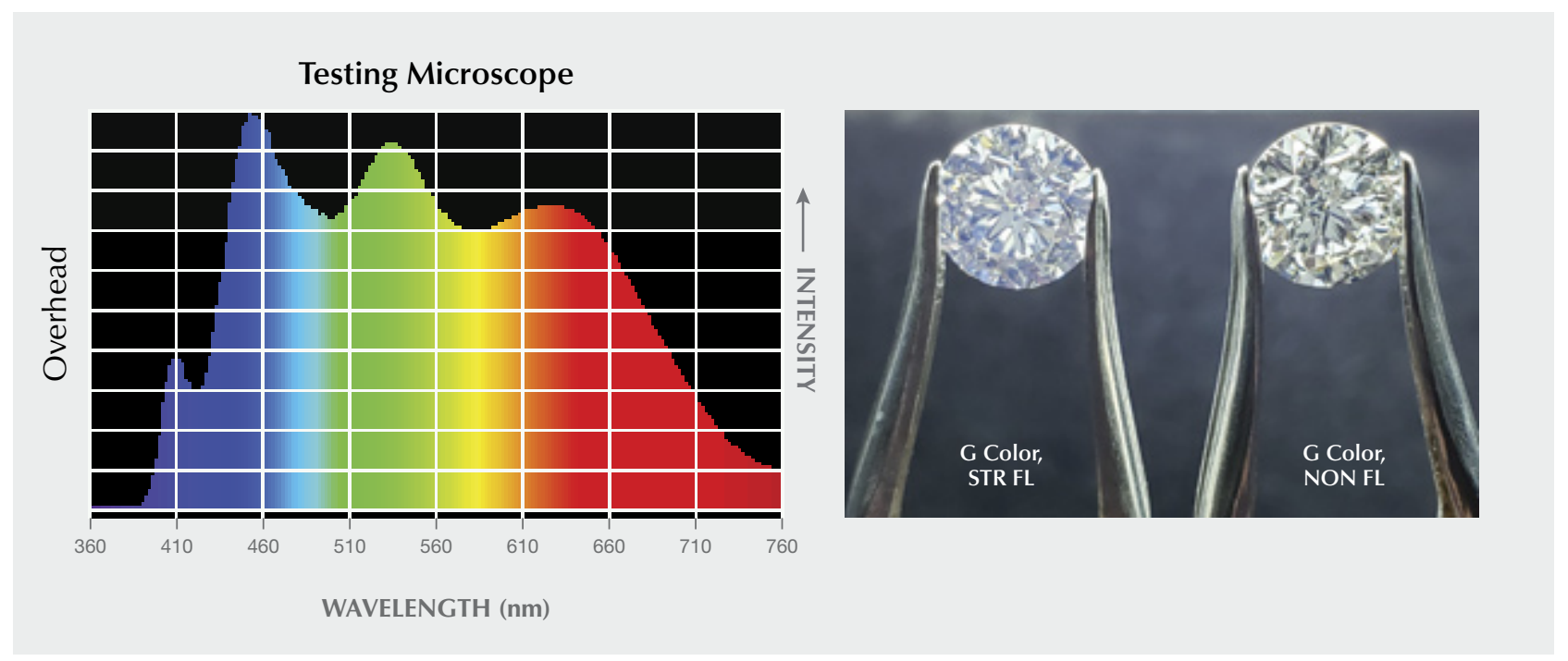




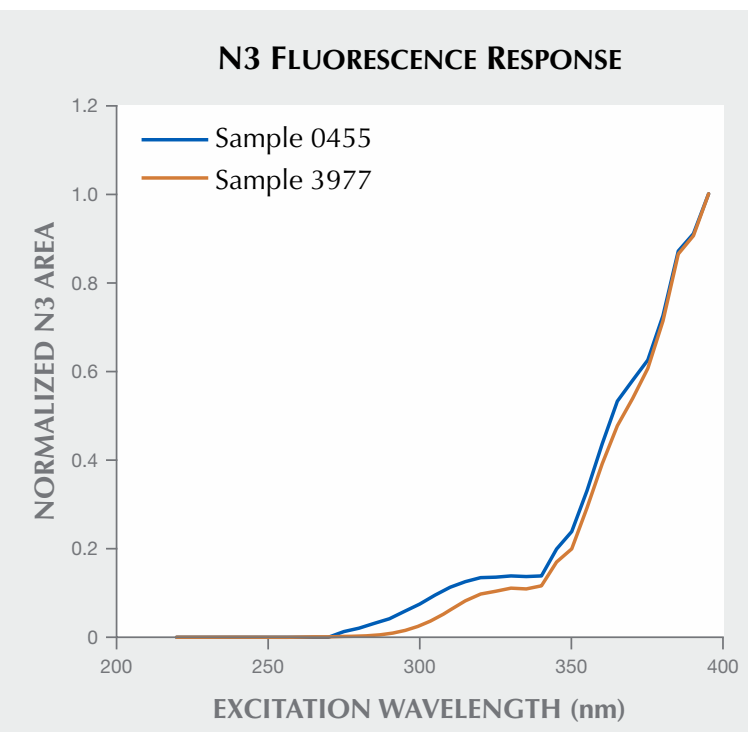

Figure 16. Two samples containing N3 were excited with narrow bands of light and the emission spectra were recorded. The area under the N3 zero-phonon line was calculated for each spectrum and plotted against its excitation wavelength, with both plots being normalized to one. Data obtained from Luo and Breeding (2013).

urement and visual observation with a fixed UV content of specific known wavelength is essential.

Fluorescence N3 Center Excitation Curve. An important metric when considering a light source is how much UV light it contains. However, quantifying the amount of UV contained in a light source is not a straightforward thing to do in a way that is useful in understanding the effect of fluorescence intensity. Different wavelengths of UV light will excite the N3 defect at different efficiencies, meaning different excitation wavelengths of a given intensity will produce different intensities of emission.

To get an idea of how the different wavelengths affect the N3, we used data from a previous study where two samples were run on a luminescence spectrometer that excited the sample with a narrow band of light and recorded the emission spectrum (Luo and Breeding, 2013). Figure 16 shows the normalized area under the N3 zero-phonon line against the excitation wavelength. We can see that from 225 to $275 \mathrm{~nm}$, there is essentially no emission for the excitation used. From 275 to about 340 nm, there is a slow increase, and then from 340 to $395 \mathrm{~nm}$ there is a rapid increase. Above $395 \mathrm{~nm}$, the excitation band overlapped too much with the N3, so accurate area measurements were not possible. The shape of these plots tracks with the previously reported photoluminescence excitation (PLE) spectrum of the 440 $\mathrm{nm}$ side band of the N3 (Luo and Breeding, 2013).

A light source used to inspect or grade diamonds may contain a distribution of UV light intensities as a function of wavelength. Each of these wavelengths will interact with the N3 defect and, based on the efficiency of absorption and fluorescence reaction, contribute varying amounts to the total visual fluorescence. If we use the area under the N3 zerophonon-line (ZPL) as a proxy for visual fluorescence intensity, we can see that the wavelength of the UV source will affect the intensity of the fluorescence. In theory, based on the chart in figure 16, we would expect a stone containing only N3 excited with a given intensity of pure $350 \mathrm{~nm}$ light to be approximately one-third as bright as the same stone excited with an equal intensity of pure $375 \mathrm{~nm}$ light.

Figure 14 shows the UV component of a number of common light sources, all normalized to the full spectrum's value at $560 \mathrm{~nm}$. If we take these spectra and multiply them by the normalized $\mathrm{N} 3$ area curves and integrate that total area, we can compare the relative fluorescence brightness we would expect from each light source. To compare different light sources, we calculated the integrated area under the normalized N3 area excitation curves (N3) multiplied by the UV component of the listed light source (normalized to $560 \mathrm{~nm}$ ) from 360 to $395 \mathrm{~nm}$. In table 5 , we see some surprising results. The expected fluorescence brightness for the table-down color measurement system with 0\% UV LED power is not 0 , but is over half the value of the system at $100 \%$ UV LED power, due to a slight UV component of the LEDs used to generate the "white light" portion of the spectrum. This is important to note because even a system labeled as a "white light" source may contribute to fluorescence.

Blue Fluorescence Effect on Color and Brightness. GIA has consistently used approximately $2 \%$ UV content in the GIA DiamondDock. Using the GIA DiamondDock as a standard for UV content (table 5), if diamonds with similar color but different fluorescence intensities are brought into an environment with higher UV content—such as outdoors under sunlight - we would not expect any observable change in color or brightness among diamonds with fluorescence descriptions of None, Faint, or 
Medium. In contrast, we should see changes in color and brightness in diamonds with Strong or Very Strong fluorescence that make them appear more colorless in sunlight. If we view these same examples in an environment with reduced UV content (such as most office lighting), the diamonds with fluorescence descriptions of None, Faint, or Medium will appear unchanged while those with Strong or Very Strong fluorescence will appear more yellow and less bright.

Blue Fluorescence Effect on Transparency. When we view a nonfluorescent diamond, we typically see a very distinct pattern with sharp boundaries between dark and light areas (figure 8 , right) created by the facet arrangement. If we look at this pattern in a diamond with Very Strong fluorescence, in a lighting environment with some UV content (figure 8, left), we see that the fluorescence makes the stone brighter; however, because the dark areas are less dark and the light areas gain a blue hue from the fluorescence, contrast is reduced. For this study, we focused on the round brilliant cut; other shapes or cutting styles were not evaluated and may be affected differently. From our MTF studies, contrast loss was measureable at line-pair frequencies less than 20 $\mathrm{lp} / \mathrm{mm}$ for plates with Medium, Strong, or Very Strong fluorescence. Five senior diamond graders at GIA were given the same micro slide used in the MTF measurements and asked to note the highest frequency of line-pairs they could resolve in a standard grading environment with a $10 \times$ loupe. The graders reported anywhere between $32 \mathrm{lp} / \mathrm{mm}$ and 48 $\mathrm{lp} / \mathrm{mm}$ as the limit of their visual resolution. Therefore, any contrast difference measured at frequencies

TABLE 5. Comparison of N3 excitation curves by various UV light sources.

\begin{tabular}{lc}
\hline \multicolumn{1}{c}{ Light Source } & $\begin{array}{c}\text { Total Area } \\
\text { Under Curve }\end{array}$ \\
\hline GIA microscope overhead light & 11.09 \\
Office light & 16.41 \\
Color measurement system with no UV & 18.18 \\
GIA microscope well light & 18.71 \\
Color measurement system with UV & 30.95 \\
GIA DiamondDock & 36.62 \\
Daylight - cloudy day & 40.22 \\
Daylight - sunny day & 73.96 \\
\hline Total integrated area $=\int_{360}^{395} \mathrm{~N} 3 \times U \mathrm{~V} d \lambda$ & \\
\hline
\end{tabular}

BLUE FLUORESCENCE IN DIAMOND less than $40 \mathrm{lp} / \mathrm{mm}$ could potentially be noticeable to a diamond grader under controlled conditions.

Several studies (Gu et al., 2019; Eaton-Magaña et al., 2019) have suggested that a milky or hazy appearance observed in Fancy white, type IaB diamonds is due to nano-inclusions or complex structural defects known as dislocation loops. Light scattering (see the Tyndall effect in box A) caused by these imperfections creates the milky or hazy appearance in these diamonds and affects their transparency. When observed with a diamond in the face-up orientation, scattering will cause a contrast loss in the facet pattern similar to that caused by fluorescence. When a diamond is strongly fluorescent and also contains light-scattering structural defects, these producers of contrast loss seemingly combine to cause a noticeably milky or hazy appearance.

Characterization of Contrast Loss Caused by Fluorescence and Haziness. Both fluorescence and light scattering cause contrast loss when looking at a diamond face-up, and it can be difficult to distinguish between their effects. We designed a bulk contrast evaluation system and explored its ability to measure contrast loss. In this evaluation, no considerations were made regarding the cause of haziness in the sample, which could be fluorescence, nano-inclusions, or dislocation loops-we only measured the contrast in the face-up pattern. The x-axis units in figure 17 are lightness, where 0 is darkest and 100 is lightest. The y-axis units describe the quantity of pixels in the image that have the corresponding lightness value. For a relatively clear diamond (top), we see the largest distance between the majority of dark pixels and the majority of the lightest pixels. As the sample gets hazier (middle and bottom), we see contrast loss (and a shrinking of this distance between the majority of the lightest and darkest pixels)—pixels that make up the dark areas in the facet pattern are lighter, and the lightest areas in the facet pattern are darker. The authors consider this bulk contrast evaluation method a relatively easy and consistent way to evaluate haziness and its impact on clarity grading of diamonds.

\section{CONCLUDING REMARKS}

Among the D-to-Z color gem diamonds that fluoresce, our results strongly suggest that the color will be noticeably impacted only in those few that show Strong to Very Strong blue fluorescence. Of course, the degree of impact depends largely on the amount 


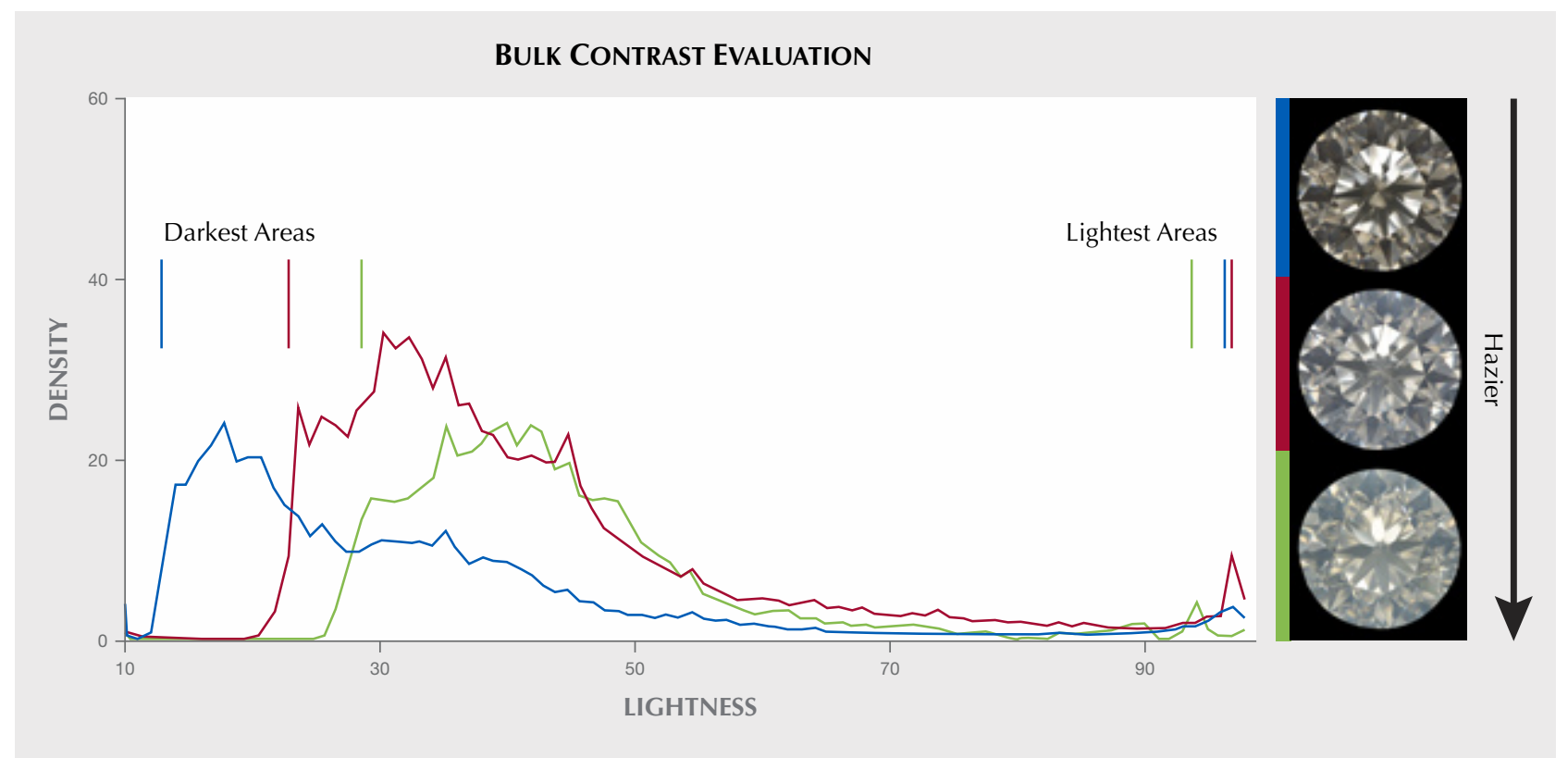

Figure 17. The bulk contrast measurement used in our study provides a relatively easy way to evaluate the face-up appearance of diamonds.

of UV component in the lighting source, meaning that a standardized lighting environment with a fixed amount of UV is critical for consistency in the color grading of diamonds. The GIA DiamondDock's UV content of $\sim 2 \%$ provides an appropriate compromise between the UV content in sunlight $13 \%$ to $5 \%)$ and that in most fluorescent and LED illumination sources used indoors (less than 1\%).

We observed that stronger fluorescence produces some contrast loss in gem diamonds. However, our results show that this contrast loss from strong fluorescence does not by itself cause the milky or hazy appearance observed in some diamonds by the trade. Atomic-scale defects in the diamond structure or nano-inclusions appear to be the main causes of the milky or hazy appearance. The occurrence of strong fluorescence in combination with these features may cause a diamond to appear even milkier or hazier, but we saw no evidence that strong fluorescence alone produces noticeable haziness in diamonds that did not already contain light-scattering structural defects or nano-inclusions. The bulk contrast method presented here may also serve as a reliable way to quantitatively evaluate the effect of contrast loss on apparent transparency in future diamond grading processes. We are reviewing these quantitative and semi-quantitative results to see how they might be included in GIA grading reports. We believe this new information will help to create more accurate information in the trade and ultimately allow consumers to select diamonds based on unbiased scientific and aesthetic factors.

\section{ABOUT THE AUTHORS}

Dr. Luo is a director of metrology and quality control, Mr. Nelson is a senior metrologist, Mr. Ardon is a research associate, and Dr. Breeding is a senior manager of analytics, at GIA in Carlsbad, California.

\section{ACKNOWLEDGMENTS}

The authors thank GIA's Tom Moses, Wuyi Wang, Tsung-Han Tsai, and Jennifer Hwang for many helpful discussions. Assistance from Mitchell Lyn and Curtis Berry in collecting image data at GIA is also greatly appreciated. The authors are grateful for the constructive comments from the three peer reviewers of this article. 


\section{REFERENCES}

ASTM (1996) ASTM Standards on Color and Appearance Measurement, 5th ed. West Conshohocken, Pennsylvania.

Bouman M., Anthonis A., Smans S., Corte K., Chapman J. (2018) The effect of blue fluorescence on the colour appearance of round-brilliant-cut diamonds. Journal of Gemmology, Vol. 36, pp. 298-315.

Cowing M. (2010) The over-grading of blue-fluorescent diamonds: The problem, the proof and the solutions. Journal of Gemmology, Vol. 32, pp. 38-51.

Eaton-Magaña S., Ardon T., Breeding C.M., Shigley J.E. (2019) Natural-color fancy white and fancy black diamonds: Where color and clarity converge. $G \oplus G$, Vol. 55, No. 3, pp. 320-337, http://dx.doi.org/10.5741/GEMS.55.3.320

Gu T., Ohfuji H., Wang W. (2019) Origin of milky optical features in type IaB diamonds: Dislocations, nano-inclusions, and polycrystalline diamond. American Mineralogist, Vol. 104, pp. 652-
658, http://dx.doi.org/10.2138/am-2019-6699

King J.M., Moses T.M., Shigley J.E., Liu Y. (1994) Color grading of colored diamonds in the GIA Gem Trade Laboratory. $G \uplus G$, Vol. 30, No. 4, pp. 220-242, http://dx.doi.org/10.5741/ GEMS.30.4.220

King J.M., Geurts R.H., Gilbertson A.M., Shigley J.E. (2008) Color grading "D-to-Z" diamonds at the GIA Laboratory. G»G, Vol. 44, No. 4, pp. 296-321, http://dx.doi.org/10.5741/GEMS.44.4.296

Luo Y., Breeding C.M. (2013) Fluorescence produced by optical defects in diamond: Measurement, characterization, and challenges. $G \uplus G$, Vol. 49, No. 2, pp. 82-97, http://dx.doi.org/10.5741/GEMS.49.2.82

Moses T., Reinitz I., Johnson M., King J., Shigley J. (1997) A contribution to understanding the effect of blue fluorescence on the appearance of diamonds. $G \uplus G$, Vol. 33, No. 4, pp. 244-259, http://dx.doi.org/10.5741/GEMS.33.4.244

\section{For online access to all issues of GEMS \& GEMOLOGY from 1934 to the present, visit:}

